\title{
Harnessing Macrophages for Controlled-Release Drug Delivery: Lessons From Microbes
}

\section{OPEN ACCESS}

Edited by:

Yurong Lai,

Gilead, United States

Reviewed by:

Daniela Giacomazza,

Italian National Research Council, Italy

Massimo Valoti,

Università degli Studi di Siena, Italy

Constantin Mircioiu,

Carol Davila University of Medicine and Pharmacy, Romania

*Correspondence:

Anton Du Preez Van Staden advstaden@outlook.com

Carine Smith

csmith@sun.ac.za

Specialty section:

This article was submitted to Drug Metabolism and Transport, a section of the journal

Frontiers in Pharmacology

Received: 19 September 2018 Accepted: 09 January 2019

Published: 25 January 2019

Citation:

Visser JG, Van Staden ADP and Smith C (2019) Harnessing Macrophages for Controlled-Release

Drug Delivery: Lessons From Microbes. Front. Pharmacol. 10:22. doi: 10.3389/fphar.2019.00022

\author{
Johan Georg Visser, Anton Du Preez Van Staden* and Carine Smith* \\ Department of Physiological Sciences, Stellenbosch University, Matieland, South Africa
}

With the effectiveness of therapeutic agents ever decreasing and the increased incidence of multi-drug resistant pathogens, there is a clear need for administration of more potent, potentially more toxic, drugs. Alternatively, biopharmaceuticals may hold potential but require specialized protection from premature in vivo degradation. Thus, a paralleled need for specialized drug delivery systems has arisen. Although cell-mediated drug delivery is not a completely novel concept, the few applications described to date are not yet ready for in vivo application, for various reasons such as drug-induced carrier cell death, limited control over the site and timing of drug release and/or drug degradation by the host immune system. Here, we present our hypothesis for a new drug delivery system, which aims to negate these limitations. We propose transport of nanoparticle-encapsulated drugs inside autologous macrophages polarized to M1 phenotype for high mobility and treated to induce transient phagosome maturation arrest. In addition, we propose a significant shift of existing paradigms in the study of host-microbe interactions, in order to study microbial host immune evasion and dissemination patterns for their therapeutic utilization in the context of drug delivery. We describe a system in which microbial strategies may be adopted to facilitate absolute control over drug delivery, and without sacrificing the host carrier cells. We provide a comprehensive summary of the lessons we can learn from microbes in the context of drug delivery and discuss their feasibility for in vivo therapeutic application. We then describe our proposed "synthetic microbe drug delivery system" in detail. In our opinion, this multidisciplinary approach may hold the solution to effective, controlled drug delivery.

Keywords: synthetic microbe, expulsion, cell-mediated delivery, biopharmaceutical, phagocytosis, intracellular pathogen, polymer, nano-medicine

\section{INTRODUCTION}

In recent years, drug delivery has become a well-documented research niche across various disciplines in science. Approaches of drug delivery into pathogenically damaged areas or poorly vascularised cancer tissues has been largely focused on treatments incorporating nanoparticles (Zhao et al., 2011; Dreaden et al., 2012; Feng et al., 2014; Huang et al., 2015; Lv et al., 2016; Tanei et al., 2016). These nanoparticles generally serve to shield harsh/labile drugs from the host and subsequently activate or release it after reaching target tissues. With the potential exception of nanoparticle uptake into target cells via complementary receptor ligands, this approach is, however, still more comparable to drug saturation than with specialized drug delivery per se. 
In this review we propose an alternative to the strategies/approaches used until now: a novel macrophage-mediated drug delivery method that more accurately fits the term "drug delivery," via incorporation of both nanomedicine and cellular manipulation. Macrophages are highly mobile cells. By loading host macrophages with appropriate "cargo" (e.g., chemotherapeutic agents such as doxorubicin or high-potency antimicrobials), one can thus theoretically use the inherent homing capabilities of these immune cells to reach target damaged, infected or malignant tissue, in order to treat the affected cellular areas only. Such an approach would reduce the total concentration of drug required (when compared to systemic administration) and significantly reduce or even eradicate the risk of drug-associated adverse effects. Achieving this goal would indeed require substantial research into phagocytosis, macrophage chemotaxis, pathogenic immune evasion and controlled release of therapeutics. Here, we propose such a system where "cargo" is introduced into the macrophage, maintained within "inactivated" phagosomes and released in a controlled manner at the appropriate time and in vivo location.

The system as proposed in its entirety here, is novel. However, some aspects of this system have been investigated individually before (discussed in detail in Section "The Impossible Made Possible?") and testifies to the feasibility of the approach we suggest. In order to fully understand cellular role players, a multidisciplinary approach is clearly required. We propose that the literature on host-microbe interactions may provide the insight required. While research has described the ability of microbes to evade the immune system by hiding (and proliferating) inside immune cells before orchestrating their own expulsion or transfer directly into new host cells, the mechanisms by which they achieve this have received very little attention by non-microbiologists. In our opinion, harnessing these microbial strategies could prove useful in the drug delivery niche. Thus, if a paradigm shift can be made to embrace the fact that host-affecting microbial mechanisms may potentially have therapeutic application, we believe that biologists could learn valuable lessons from microbes, to the benefit of technological advancement in medicine.

The aim of this paper is therefore to present a summary of pertinent literature on microbial mechanisms known to modulate the course of endocytic processes and to evaluate their feasibility in the context of therapeutic drug delivery. A specific novel focus will be on potential mechanisms through which to achieve controlled expulsion. We believe that this paper elucidates an exciting new avenue for research in the context of drug delivery.

In order to facilitate clarity of our argument, we first provide a brief overview of the most pertinent literature describing the mechanisms that would come into play in a complete cell-based delivery system. Considering the complexity of these processes, one can appreciate the enormity of the task to elucidate which perturbations in this process may be used for application to our proposed drug delivery system. Thus, we will describe the different phases - namely cargo loading, maintenance of cargo integrity, in vivo motility of the carrier cell toward delivery sites and cargo expulsion - individually below, before discussing in more detail, the lessons to be learnt from microbes.

\section{COMPONENTS OF A CELL-BASED DELIVERY SYSTEM}

\section{Cargo Loading Into Macrophages}

Circulating monocytes form part of the innate immune system and are largely responsible for the initial recognition of foreign material or microbes (Abbas et al., 2014). Recognition and internalization, for the purpose of neutralization, are generally very effective. This is evidenced by the absence of adaptive B and $\mathrm{T}$ cell responses in almost 95\% of Animalia (Mills et al., 2015). However, many microbes have been able to survive within macrophages by manipulating phagocytic processes (discussed later in Section "What Can We Learn From Microbes?"). A summary of the most relevant normal human phagocytic processes is presented visually in Figure 1.

The most important aspect of our topic, is that of immune recognition and uptake into the macrophage. It is commonly known that pattern recognition receptors (PRRs) on phagocytes recognize several different molecular patterns - such as damageassociated molecular patterns (DAMPs) or pathogen-associated molecular patterns (PAMPs) - on the target for potential phagocytosis (Abbas et al., 2014). Toll-like receptors (TLRs) present on phagocytes also indirectly regulate phagocytosis through Myeloid differentiation primary response 88 (Myd88) signaling and activation of the p38 residue (Shi et al., 2016). Several other minor role players in pathogen recognition, such as receptors for lectin, mannose, complement and Retinoic acidinducible gene-I-like (RIG-like) receptors, have been identified, but the immunoglobulin $\mathrm{G}$ (IgG) receptors are most directly associated with phagocytosis of material. In fact, antibodyopsonised material binds and activates IgG receptors to induce engulfment independently of co-stimulation by $\mathrm{T}$ cells or $\mathrm{NK}$ (natural killer) cells (Liu et al., 2013), making this mechanism an obvious choice for ex vivo cargo loading into macrophages. Engulfment is reliant on phosphoinositide 3-kinase (PI3k) recruitment and its production of various phosphatidylinositides that, together with actin polymerization, result in pseudopod formation around the material and subsequent internalization.

Once material has been engulfed, it is enveloped inside a double-membraned (nascent) phagosome, which is innocuous and undergoes various maturation phases, that culminates in fusion with lysosomes, which enables it to acidify and break down its contents. Characterization of the engulfment and early maturation processes is well-established (Patki et al., 1998; Fratti et al., 2001; Vieira et al., 2002, 2003; Kinchen et al., 2008; Fairn and Grinstein, 2012) and are not discussed in more detail here, as we do not envisage a requirement for huge manipulation of this phase. Indeed, previous research by our group and others have demonstrated that macrophages readily take in a variety of purpose-designed materials and particles of varying sizes via endocytic pathways (Muthana et al., 2011; Zhao et al., 2011; 


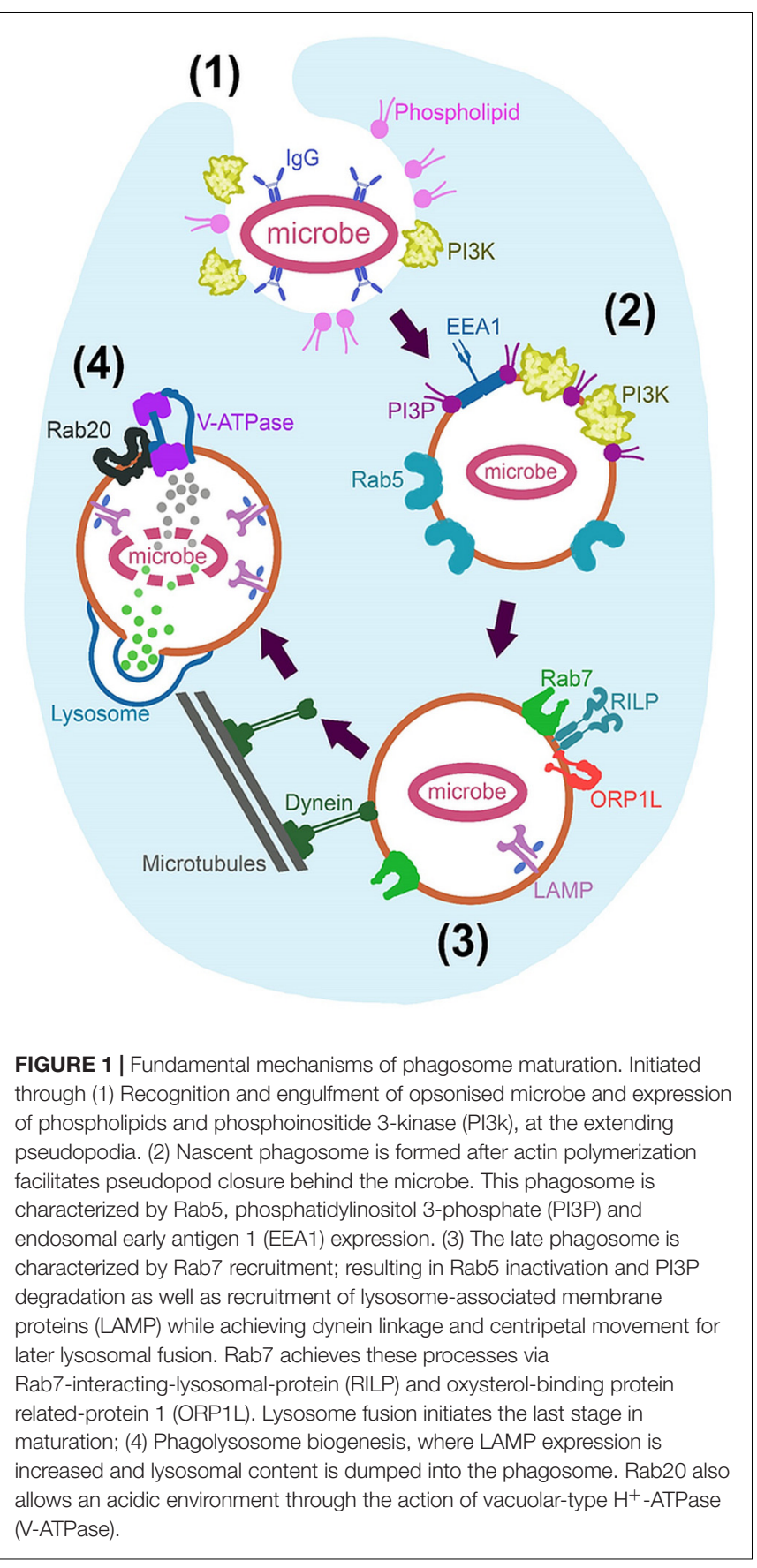

Dreaden et al., 2012; Feng et al., 2014; Oh and Park, 2014; Huang et al., 2015; Miller et al., 2015; Tanei et al., 2016; Fan et al., 2018; Visser and Smith, 2018).

\section{Cargo Maintenance}

Of more direct relevance, lysosomal fusion marks the start of the last stage in maturation, that of phagolysosome biogenesis (Seto et al., 2011), which is an obvious threat to cargo maintenance. Normally, this lysosome fusion is mediated by endoplasmic reticulum (ER) soluble $N$-ethylmaleimide-sensitive factor-attachment proteins (SNARE) such as syntaxin 7 , syntaxin
8 and vesicle associated membrane protein (VAMP) -7 and -8 (Becken et al., 2010). Lysosome-associated membrane protein (LAMP) concentration is increased after fusion (Jahraus et al., 1994) and cathepsin D proteases are recruited from the Golgi via Rab-22b, -32, -34, -38, and -43 (Ng et al., 2007). The vacuolar-type $\mathrm{H}^{+}$-ATPase (V-ATPase) is also incorporated via Rab20 co-localization at this time (Curtis and Gluck, 2005). In this way, fusion ultimately effectuate an acidic environment within the macrophage phagosome, as well as supplying it with proteases, reactive oxygen species (ROS) and reactive nitrogen species (RNS) to facilitate decomposition of phagosomal content.

To date, the majority of literature employing macrophages as delivery shuttles, have used either nanoparticle-encapsulated drugs traveling inside the cell, or drugs "backpacked" on the outside of the cell. The most popular protocol used is loading cargo into macrophages to create a "Trojan horse." However, this approach has some limitations: firstly, there is a significant risk of drug-associated cytotoxicity, secondly, drugs are released at a relatively slow rate and thirdly, they are vulnerable to lysosomal degradation inside the macrophage (Yousefpour and Chilkoti, 2014). In an attempt to address these limitations, transport of drugs on the outer surface of macrophages were attempted. However, prevention of internalization of the backpacked cargo into carrier macrophages was a major obstacle (Holden et al., 2010; Doshi et al., 2011).

In our opinion, perhaps the most feasible option to ensure integrity of cargo that are either labile or highly toxic - so that premature delivery should not be risked - would be their maintenance intracellularly by modification of normal phagocyte function. It is here where we could substantially learn from microbial strategies (refer to Section "What Can We Learn From Microbes?"). Indeed, we have previously demonstrated maintenance of cargo inside primary human M1 macrophages chemically treated to transiently inhibit phagosomal cargo destruction (Visser and Smith, 2018). Briefly, protein-coated polystyrene beads, used as simulative cargo, were maintained intact (i.e., with no digestion of the protein coating) inside macrophages after in vitro treatment with a phagosome maturation inhibiting cocktail, consisting of Wortmannin, Concanamycin A and Chloroquine. This inhibition cocktail was only administered in vitro, and treated cells were washed prior to use, thus lowering risk to patient in the context of in vivo application. Furthermore, this intervention did not affect chemotactic or migratory capacity - macrophages were able to transverse an in vitro Human Umbilical Vein Endothelial Cell (HUVEC) membrane while carrying the bead cargo.

Another modern technique relevant here, is the use of nano- or microparticle encapsulation of drugs prior to loading into carrier cells (Dou et al., 2006; Zhao et al., 2011; Chang et al., 2013; Blaudszun et al., 2014; Feng et al., 2014; Gnanadhas et al., 2017; Klyachko et al., 2014; Pang et al., 2016; Tanei et al., 2016; Evans et al., 2018; Fan et al., 2018). In addition to host protection, polymeric particles may also be used for maintenance of drug integrity itself. Emerging evidence indeed indicates a role for polymeric particles as protective modality for both host and drug cargo. Cargo can be rendered innocuous via, for example, poly-(NIPA-co-AAm) (PNIPAAm) micelle or 
microbubble encapsulation. PNIPAAm micelles are reported to degrade in response to an increase in temperature above the lower critical solution temperature (Feng et al., 2014), enabling control over bio-activation of encapsulated drugs. As an example, these micelles could be incorporated during treatment of peripheral diseases, such as melanoma or myopathy, where an external stimulus can be administered to increase the local temperature and release drug cargo from PNIPAAm micelles. Incorporation of microbubbles together with nanoparticles has also shown some promise during in vivo delivery of resveratrol for treatment of cancer (Lv et al., 2016). In this study, resveratrol was loaded into acetylated $\beta$-cyclodextrin nanoparticles (PNP), which were then loaded into microbubbles. The outer microbubble coating served to protect the $\mathrm{pH}$ sensitive PNP while in circulation, whereas PNP in turn released resveratrol upon exposure to the low $\mathrm{pH}$ tumor niche. These studies indicate that polymeric particles may be powerful tools to incorporate into delivery systems to address current limitations.

\section{In vivo Macrophage Migration for Cargo Delivery}

Literature focusing on macrophage (or other phagocyte) migration are normally aimed at the prevention of this migration, e.g., in the context of cancer metastasis or inflammation. Despite the different focus to ours, these studies have elucidated the process of migration in detail.

For example, in the context of muscle inflammation, M1 macrophages have been illustrated to be the most motile, pro-inflammatory phenotype, while M2 macrophages are less likely to infiltrate tissues and associated with a relatively antiinflammatory outcome (Arnold et al., 2007; Smith et al., 2008; Kruger et al., 2014). Our previous work on M1 macrophages (Visser and Smith, 2018) confirms the choice of this phenotype as superior for drug delivery. However, it should be noted that macrophage phenotype has a large degree of plasticity, which will have to be taken into account when designing drug delivery protocols for application in particular disease states. To this end, a recent review (Ruytinx et al., 2018) comprehensively provide information on macrophage polarization in the context of inflammatory diseases such as neurological disease, cancer, metabolic and cardiovascular disorders.

Similarly, in terms of chemotactic signals for macrophage migration, chemotaxins generally expressed on tissue cells in many different disease conditions have been identified. Most notably, inflammation - which would be present in any disease state with a requirement for drug delivery - is known to result in increased levels of the chemokines macrophage migration inhibitory factor (MIF) and/or macrophage chemotactic protein (MCP-1 or CCL2), which are strong signals for macrophage recruitment into the tissue (Lee et al., 2010; Baeck et al., 2012). Additionally, oxidative stress - which is a known complication of both chronic disease and infection (Nimse and Pal, 2015; Petersen and Smith, 2016) - have been shown to initiate macrophage migration in vivo (Wang et al., 2016). In terms of systemic migration toward chemotactic signals originating from hypoxic tissue, such as in cancer, evidence also exist to confirm the inherent capacity of macrophages to migrate toward tumors (Owen et al., 2004; Batrakova et al., 2011). Although finer detail on the regulation of macrophage migration has been reported, such as its dependence on integrin $\beta 1$ expression and recycling (Lee et al., 2017) and numerous proteases (Van Goethem et al., 2018), these details are likely of academic value only, at least for the context under discussion. In our opinion, these factors are unlikely to be a limiting factor, since integrin $\beta 1$ is expressed by almost all cell types and some degree of redundancy is in place. For example, in contrast to the fairly uniform amoeboid movement of neutrophils, macrophages were reported to exhibit multiple different migration mechanisms that are more mesenchymal in nature (Barros-Becker et al., 2017; Van Goethem et al., 2018), which could confer greater resilience to macrophages in terms of mobility under adverse conditions. Interestingly, the latter study also demonstrated a larger degree of directionality of macrophages $v s$. neutrophils in a zebrafish tail transection model of leukocyte migration. Another ability of macrophages pertinent to the current topic, is their ability to maintain their mobility after ingestion of cargo, even when the cargo is much larger than anticipated to be required for the purpose of drug delivery (Chang et al., 2013; Evans et al., 2018; Visser and Smith, 2018). These reports again confirm that this phase of the system is unlikely to require major intervention, as it seems to already have been fine-tuned by evolution.

The only potential limitation we foresee is interference with chemotactic signal originating from the intended site for drug delivery, by e.g., an acute, severe infection/damage in another organ, which may have chemotactic priority above that of the signal originating from the intended delivery site. However, the practice of isolating patients for a period prior to a medical procedure is not uncommon and could avoid this complication. Furthermore, pathogen-associated infection has been shown to take priority above other, relatively less life-threatening, situations, which would in fact favor directional macrophage migration, rather than limit it.

\section{Cargo Expulsion}

The final step to complete such a delivery system, would be a mechanism by which the cargo can be released or expelled at the appropriate time and location in vivo. Normally, following phagolysosomal destruction of ingested material, digested material is either recycled by the phagocytic cell or expelled into the extracellular matrix. Recycling of re-usable "waste" such as amino acids, glucose and phosphates occur via diffusion through the phagolysosome membrane into the cytosol (Guyton and Hall, 2011). Of particular interest here, the insoluble components are expelled from the macrophage either via the ER-Golgi secretory pathway or utilized for antigen presentation through $\mathrm{Ca}^{2+}$ - and vesicle-associated membrane protein 7 (VAMP7)-dependant lysosome exocytosis (Samie and $\mathrm{Xu}, 2014)$. We believe that the manipulation of these expulsion mechanisms could facilitate controlled drug delivery.

In terms of published studies on drug delivery systems, most systems either rely on non-specific release of nanoparticles containing drugs (Miller et al., 2015), or employ release of drugs inside the carrier cell. For example, rupture of 
doxorubicin-containing microbubbles inside macrophages was achieved by high intensity focused ultrasound techniques (Fan et al., 2018). However, this strategy for drug release resulted in significant carrier cell death. We believe this is an undesirable mechanism, as this would likely contribute to inflammation and thus delayed recovery. Therefore, to date, a controlled cell-based drug release system does not seem to exist.

In the next section, we evaluate microbially employed strategies, in terms of their feasibility for adaptation into therapeutic contexts. We will focus on the two phases of this system which seems to be most commonly and effectively manipulated by microbes, namely immune evasion by intracellular survival and programmed expulsion from host cells.

\section{WHAT CAN WE LEARN FROM MICROBES?}

Pathogenic phagosome maturation arrest or modulation thereof, and subsequent excape from the host cell are hallmarks of bacterial host immune evasion and dissemination. Well characterized mechanisms include interfering with PI3k and PI3P biogenesis (Mycobacterium tuberculosis and Candida glabrata) (Vergne et al., 2003; Rai et al., 2015), establishing microbe-containing vacuoles (Legionella pneumophila and Brucella) (Celli, 2015; Bärlocher et al., 2017), blocking of fission and fusion with lysosomes and endosomes (Mycobacterium tuberculosis and Legionella) (Vergne et al., 2003, 2005; Bärlocher et al., 2017), raising $\mathrm{pH}$ levels via induction of phagosomal acid leakage (Cryptococcus neoformans) (Tucker and Casadevall, 2002), lysis of the phagosomal membrane (Listeria monocytogenes) (Alberti-Segui et al., 2007), hijacking of the endocytic recycling pathway (Legionella pneumophila) (Xu and Luo, 2013) and even active macrophage killing (filamentous Candida albicans) (Gaur et al., 2013). Manipulation of the endocytic pathways by microbes is achieved via highly diverse and complex mechanisms. Intracellular microbes secrete hundreds of proteins, known as effectors, capable of modulating these pathways (Santos et al., 2015; Schroeder, 2018). These effectors have diverse functions and microbes employ multiple layers of redundancy to ensure their survival (Ghosh and O'Connor, 2017; Schroeder, 2018). The abundance and variety of these effectors provide an ideal bioprospecting opportunity to identify effectors that can be utilized to modulate the endocytic pathways as needed. Keeping in mind that not all microorganisms have effectors capable of manipulating the endocytic pathway for both maintenance and expulsion. Identified effectors can then be further investigated to optimize the cocktail of effectors (possibly from different organisms) best suited for application in a macrophage-based delivery system.

Examples of intracellular microbes and their mechanisms for modulation of the endocytic pathway are summarized in Table 1. In order to provide more detail on the variety and complexity of methods used, modulatory mechanisms of different microbes in the context of both phagosome maturation and expulsion are presented in the next sections.

\section{Intracellular Survival Mechanisms}

Due to the high incidence of tuberculosis in especially developing countries, much research has been focused on the causative agents of this illness. As a result, relatively detailed knowledge is available on the route of immune evasion by this pathogen in particular, as well as on how bacterially secreted effectors and cell wall components modulate phagosome maturation. The primary route of Mycobacterium tuberculosis (Mtb) into the body is through inhalation, where it reaches the lungs' alveolar space and is preferentially taken up by alveolar macrophages. Mtb survive intracellularly by working against PI3ks to prevent EEA1 docking. This is achieved in two ways: (1) Mtb secretes a PI 3 -phosphatase (SapM) that dephosphorylates PI3P and (2) a component in the microbial cell wall, lipoarabinomannan (LAM), interferes with recruitment/activation of the human PI3k (hVPS34) (Vergne et al., 2005). Mycobacterium-containing phagosomes also retain the tryptophan-aspartate containing coat (TACO) protein (normally expressed on the cytosolic leaflet of the plasma membrane and involved in intracellular membrane trafficking, cytokinesis and cytoskeletal remodeling) (Ferrari et al., 1999). TACO retention causes prolonged Rab5 expression - although some maturation effectors can still bind the phagosome, this effectuates a relative absence of PI3P, so that the FYVE domain-mediated binding of EEA1 is greatly perturbed (Simonsen et al., 1998) and lysosome fusion inhibited (Ferrari et al., 1999). Additionally, secretion of the soluble serine/threonine kinase Protein kinase G (PKG) by Mtb into the host cytosol is essential for prevention of phagosome-lysosome fusion (Walburger et al., 2004). Furthermore, a more alkaline and hydrolase deficient phagosome is also brought about in two ways. Firstly, hydrolysis is weakened by limited expression of Rab7. This GTPase has been shown to only transiently localize to mycobacterial phagosomes, preventing sufficient Rab7-interacting lysosomal protein (RILP) recruitment, but also limiting cathepsin D protease delivery (Seto et al., 2010, 2011). Secondly, acidification is regulated by Mtb by interfering with V-ATPase complex assembly and retention, thereby maintaining a stable, slightly alkaline $\mathrm{pH}$ (6.2-6.5) (Sturgill-Koszycki et al., 1994; Seto et al., 2011; Queval et al., 2017). The Mtb phosphatase, PtpA, is involved in inhibition of complex assembly by binding to the subunit $\mathrm{H}$ of the V-ATPase where it then dephosphorylates and inactivates hVPS33B, effectively inhibiting the membrane fusion machinery (Wong et al., 2011).

In contrast to $\mathrm{Mtb}$, the survival mechanisms of C. glabrata is largely dependent on active PI3ks. C. glabrata encodes the enzyme PI3k and produces fungal PI3P (Strahl and Thorner, 2007; Rai et al., 2015). In this manner, the PI3P content of phagosomes increase prematurely during the early stages of maturation, where PI3P has not yet come into play. This could lead to a PI3P rich phagosome being identified as already partly matured, thus halting further maturation. Additionally, increased PI3P content could overburden PI3P degradation capacity of the phagosomal lumen. Deletion of the functional subunits of fungal PI3k led to ameliorated phagosome maturation and 
TABLE 1 | Examples of intracellular microbes and main outcomes of endocytic pathway modulation.

\begin{tabular}{|c|c|c|c|c|}
\hline Organism & Disease state & $\begin{array}{l}\text { Type of endocytic } \\
\text { modulation }\end{array}$ & $\begin{array}{l}\text { Outcome of endocytic } \\
\text { modulation }\end{array}$ & $\begin{array}{l}\text { Egress and } \\
\text { dissemination }\end{array}$ \\
\hline $\begin{array}{l}\text { Brucella (G-) } \\
* 1\end{array}$ & Brucellosis/Malta Fever & $\begin{array}{l}\text { Modulation of phagosome } \\
\text { maturation }\end{array}$ & $\begin{array}{l}\text { VirB (T4SS) dependent } \\
\text { modulation of phagosome, } \\
\text { Suppression of } \\
\text { macrophage polarization }\end{array}$ & $\begin{array}{l}\text { Cell necrosis/apoptosis } \\
\text { (VirB and bacterial } \\
\text { dissociation dependent) } \\
\text { followed by extracellular } \\
\text { dissemination }\end{array}$ \\
\hline $\begin{array}{l}\text { Legionella pneumophila (G-) } \\
* 2\end{array}$ & Legionnaires disease & $\begin{array}{l}\text { Prevention of phagosome } \\
\text { maturation }\end{array}$ & $\begin{array}{l}\text { Dot/lcm (T4BSS) } \\
\text { dependent prevention of } \\
\text { phagosome maturation }\end{array}$ & $\begin{array}{l}\text { Pyroptosis, Apoptosis, Cell } \\
\text { lysis }\end{array}$ \\
\hline $\begin{array}{l}\text { Listeria monocytogenes } \\
(\mathrm{G}+) \\
*_{3}\end{array}$ & Listeriosis & $\begin{array}{l}\text { Prevention of phagosome } \\
\text { maturation, phagosome } \\
\text { rupture }\end{array}$ & $\begin{array}{l}\text { Listeriolysin-O dependent } \\
\text { lysis of phagosome or } \\
\text { induction of autophagy for } \\
\text { replication }\end{array}$ & $\begin{array}{l}\text { Cell-to-cell spread } \\
\text { (ActA/LLO-dependent) }\end{array}$ \\
\hline $\begin{array}{l}\text { Chlamydia (G-) } \\
* 4\end{array}$ & Genital/respiratory infections & $\begin{array}{l}\text { Subversion of endocytic } \\
\text { pathway via inclusion } \\
\text { formation }\end{array}$ & $\begin{array}{l}\text { Inc/CT229 dependent } \\
\text { inclusion development }\end{array}$ & $\begin{array}{l}\text { Cell lysis, Inclusion } \\
\text { extrusion }\end{array}$ \\
\hline $\begin{array}{l}\text { Mycobacterium } \\
\text { tuberculosis (acid fast) } \\
* 5\end{array}$ & Tuberculosis & $\begin{array}{l}\text { Prevention of phagosome } \\
\text { maturation, phagosome } \\
\text { rupture }\end{array}$ & $\begin{array}{l}\text { LAM/SapM dependent } \\
\text { interference of PI3k and } \\
\text { PI3P biogenesis. Inhibition } \\
\text { of H+ V-ATPase assembly. } \\
\text { ESX-1/EsXA and PDIM } \\
\text { dependent phagosome } \\
\text { rupture }\end{array}$ & Cell necrosis/apoptosis \\
\hline $\begin{array}{l}\text { Salmonella enterica (G-) } \\
* 6\end{array}$ & Salmonellosis & $\begin{array}{l}\text { Modulation of phagosome } \\
\text { maturation }\end{array}$ & $\begin{array}{l}\text { Modulation of phagosome } \\
\text { maturation via T3SS } \\
\text { effector (SifA, SopB) } \\
\text { dependent development of } \\
\text { SCV }\end{array}$ & Pyroptosis \\
\hline $\begin{array}{l}\text { Cryptococcus neoformans } \\
\text { (Yeast) } \\
* 7\end{array}$ & Cryptococcal meningitis & $\begin{array}{l}\text { Unknown effectors. } \\
\text { Possible pH dependent } \\
\text { phagosome damage. } \\
\text { Capsular protection. }\end{array}$ & $\begin{array}{l}\text { Unknown effectors. } \\
\text { Possible pH modulation } \\
\text { through phagosome } \\
\text { damage. }\end{array}$ & $\begin{array}{l}\text { Non-lytic expulsion, cell } \\
\text { lysis, cell-to-cell spread }\end{array}$ \\
\hline
\end{tabular}

Gram negative (G-), Gram positive (G+). *1: Pizarro-Cerdá et al., 1998; Hong et al., 2000; Comerci et al., 2001; Boschiroli et al., 2002; Celli et al., 2003, Celli, 2006, 2015; Arellano-Reynoso et al., 2005; Pei et al., 2008, 2006, 2014; Starr et al., 2008, 2012; Chen and He, 2009; Chen et al., 2011; Von Bargen et al., 2012; Smith et al..

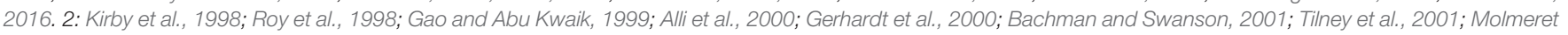

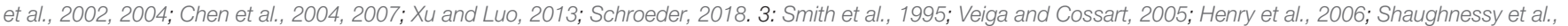

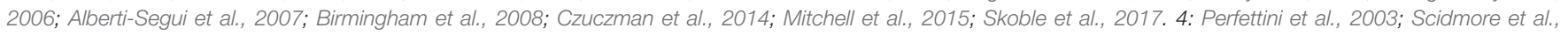

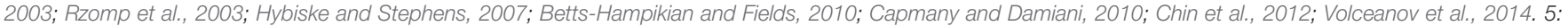

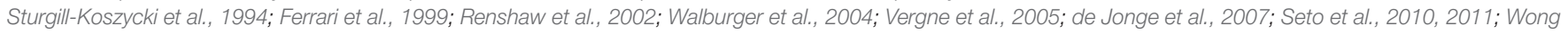

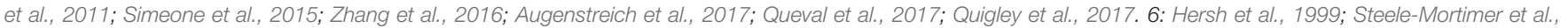

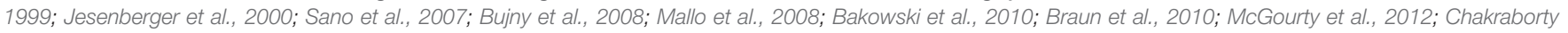

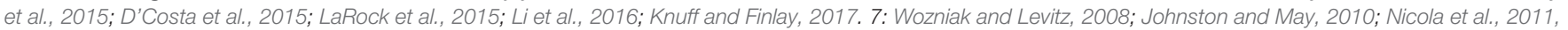
2012; Qin et al., 2011; Chen et al., 2015; Davis et al., 2015; Smith et al., 2015; Bojarczuk et al., 2016; Gillbert et al., 2017.

significantly reduced fungal survival and virulence (Rai et al., 2015). The differences between the strategy of C. glabrata vs. Mtb illustrates how the same cellular role players may be modulated in different ways for different outcomes, depending on the intended requirement of the modulating microbe, and in our opinion also demonstrates the susceptibility of this system to exogenous modulation or control.

Reminiscent of Mtb, Leishmania (the causative agent of Leishmaniasis) promastigotes are also harbored in phagosomes that retain TACO on their membranes, blocking lysosome fusion and ensuring a neutral $\mathrm{pH}$ in which this parasite can differentiate into the amastigote stage (Ferrari et al., 1999; Gogulamudi et al., 2015). However, after differentiation, the parasite allows phagosome fusion with lysosomes to achieve an acidic environment in which the amastigotes thrive. Interestingly, these phagosomes still exhibit low expression of late phagosomal markers (LAMP, V-ATPase and Rab7), after lysosome fusion (Vinet et al., 2009). In addition, Leishmania protects itself by inhibiting recruitment of NADPH oxidase to the phagosome, perturbing ROS production (Moradin and Descoteaux, 2012). Similarly, M. tuberculosis was reported to stimulate release of TNF- $\alpha$ and IL-10 from infected macrophages (Sendide et al., 2005), resulting in a deactivation of ROS and RNS release (Redpath et al., 2001). IL-10 specifically down-regulates secretion of pro-inflammatory cytokines (Redpath et al., 2001) like INF- $\gamma$ and TNF- $\alpha$ and results in a shift toward a Th2-type cell expansion in the alveoli (de Almeida et al., 2012), bringing about a shift toward an alternatively activated, anti-inflammatory, M2 macrophage phenotype (Smith et al., 2008), which itself produces more IL-10, sustaining this phenotype and a relatively more anti-inflammatory environment. This implies that these microbes not only alter the response of the host cell to the ingested microbe 
itself, but that it may also affect systemic signaling by the host cell, which may affect the rate at which these bacteria are able to spread.

Brucella and Legionella are examples of intracellular pathogens that manipulate the endocytic pathway to create a niche in which they can replicate and thrive. They accomplish this by hijacking host proteins and membrane organelles to establish a bacterium-containing vacuole with morphological features reminiscent to that of host membrane compartments ( $\mathrm{Xu}$ and Luo, 2013; Celli, 2015). Effectors secreted by Brucella within the Brucella-containing vacuole (BCV) manipulates maturation by altering interactions with late endosomes and lysosomes (Celli, 2015). During initial phagocytosis a large portion of Brucella cells are rapidly degraded ( $90 \%)$, however, surviving cells are capable of prolonged intracellular proliferation (Von Bargen et al., 2012). Previously it was thought that Brucella evade fusion of BCVs with lysosomes by secretion of effectors via a functional VirB type IV secretion system (T4SS) and cyclic $\beta$-1-2-glucan (Pizarro-Cerdá et al., 1998; Celli et al., 2003; Arellano-Reynoso et al., 2005). Cyclic $\beta$-1-2-glucan was thought to prevent fusion of the BCV with lysosomes by modulating lipid raft organization on phagosome membranes, but is not a requirement for subsequent BCV maturation (Arellano-Reynoso et al., 2005). Rather, live cell imaging has shown that the BCV interacts with lysosomes, thus fusion is not completely prevented (Starr et al., 2008). Early stages of BCV maturation involve the recruitment of late endosome markers, LAMP-1 and Rab7 to the BCV membrane, with acidification of the BCV being crucial for VirB expression (Boschiroli et al., 2002; Starr et al., 2008). This early BCV is also known as the endosomal BCV (eBCV) due to its interaction with the endocytic pathway. These findings highlight the importance of the initial interactions with the endocytic pathway in determining outcome. Unlike Brucella, Legionella diverts from the canonical endocytic pathway soon after being phagocytosed (Tilney et al., 2001). Departure from the canonical endocytic pathway starts minutes after being phagocytosed - the Legionella-containing vacuole (LCV) is covered with smooth vesicles, ER in origin, and mitochondria is recruited to the LCV (Tilney et al., 2001). The LCV is also devoid of early endosome markers such as Rab5 and LAMP-1, with the exception of Rab7 (Roy et al., 1998). L. pneumophila utilizes early mild caspase-3 activation to prevent lysosome fusion by cleavage of rabaptin5 (effector of Rab5) (Gao and Abu Kwaik, 1999; Molmeret et al., 2004). The eBCV and LCV both eventually interact with components of the ER. In the case of the eBCV, LAMP-1 is progressively lost as the eBCV interacts with the ER and maturation proceeds to the formation of a replication-permissive BCV (rBCV) (Celli et al., 2003; Celli, 2006; Starr et al., 2008), with the rBCV subsequently converted from an intermediate vacuole into an ER-derived organelle which is ideal for bacterial proliferation (Celli et al., 2003). The smooth vesicles recruited to the LCV early on, eventually come to resemble rough ER and become studded with ribosomes (Gerhardt et al., 2000; Tilney et al., 2001). The specific recruitment of GTPases usually required for fusion of ER-derived vesicles with the Golgi apparatus aids in this process. Similar to Brucella, the hijacking of the host's secretory trafficking pathway results in a replication-permissive
LCV. The rapid formation of an ER-like LCV and subversion of the endocytic pathway is dependent on the Dot/Icm T4BSS (Defective in Organelle Trafficking/Intra-Cellular Multiplication Type 4B Secretory System) of Legionella (Roy et al., 1998). Indeed, mutants deficient in the T4BSS ultimately fuse with the lysosome, indicating that effectors secreted by the T4BSS directly influence the endocytic pathway (Roy et al., 1998; Molmeret et al., 2004; Schroeder, 2018). The Dot/Icm T4BSS secretes hundreds of potential virulence effector molecules that aid in the formation of the LCV. However, no one effector has been shown to be crucial, again indicative of multiple layers of redundancy (Schroeder, 2018). Similarly, the VirB T4SS is essential for Brucella survival, as illustrated in virB-mutants (Hong et al., 2000; Comerci et al., 2001; Celli et al., 2003; Pei et al., 2008). Several other pathogens utilize T4SS and other secretion systems to release effector molecules that are capable of manipulating host function. The VirB and Dot/Icm systems are certainly also capable of releasing effector molecules that, in the case of Brucella and Legionella, are used to manipulate ER membrane dynamics and fusion.

Similar to some vacuole-inhabiting bacteria, Chlamydia also subverts the endocytic pathway to create a replicative niche. Chlamydia is also a very proficient modulator of the host cytoskeleton through complex interactions of its secreted effectors with the host cell. This manipulation is even more interesting when considering that Chlamydia has a relatively small genome for bacteria (1.04 and $1.23 \mathrm{Mb}$ for C. trachomatis and C. pneumoniae respectively) and relies on the host for their metabolic requirements (Stephens et al., 1999). Furthermore, $\sim 10 \%$ of its genome encodes for virulence effectors (Betts-Hampikian and Fields, 2010) which, as for some other intracellular pathogens, are delivered through specialized secretion systems. Similar to Legionella, the Chlamydia-containing inclusion (the term used for the replicative vacuole) is diverted from the endocytic pathway early on, and is rather trafficked to the microtubule organizing centre (MTOC) via dynein-mediated movement. From here, they are in an ideal position to intercept lipids and nutrient-rich exocytic vesicles. Markers for early endocytic- and late endocytic-compartments are absent from the inclusion (such as Rab5, Rab7 and LAMP-1) (Rzomp et al., 2003; Scidmore et al., 2003). However, several other Rab GTPases are recruited to the inclusion, such as Rab1, -4, -6 (C. trachomatis only), -10 (C. pneumoniae only), -11 and -14 (Rzomp et al., 2003; Capmany and Damiani, 2010). The recruitment of the different Rab GTAPases is important for the modulation of fusion events, for example the prevention of lysosomal fusion and promoting of fusion with lipids and nutrient-rich exocytic vesicles. Chlamydia further modulate vesicle fusion via interaction with SNARE proteins.

Subversion of the canonical phagocytic pathway by Salmonella uses similar mechanisms to that of, both, the vacuole-residing bacteria and those opting for a cytosolic lifestyle. After internalization, Salmonella remains in a modified phagosome the Salmonella containing vacuole (SCV). Similar to the microbes already mentioned, Salmonella utilize secretory systems to deliver their effectors to the host and have two T3SS encoded on different pathogenicity islands (SPI-1 and SPI-2) (LaRock et al., 2015). The early effectors secreted by Salmonella (via T3SS-SPI1) are 
important for the establishment of this early SCV. Shortly after being phagocytosed SCV associates with early endosome markers EEA-1 and Rab5 and via its effector SopB (a phosphatase), delays lysosome fusion by indirectly preventing Rab GTPases from binding to the phagosomal membrane (Steele-Mortimer et al., 1999; Mallo et al., 2008; Bakowski et al., 2010). Recruitment of sorting nexins (SNX) help in the progression of SCV maturation, SNX1 specifically induces tubulation and is involved in the removal of the cation-dependent mannose-6-phosphate receptor (MPR) that may be important for the lack of lysosomal enzymes in the late SCV (Bujny et al., 2008). Additionally, SNX3 transiently interacts with the early SCV and is required for tubule formation and recruitment of late endosomal markers Rab7 and LAMP-1 (Braun et al., 2010). The replacement of early markers at this stage is accompanied by a decrease in both bacterial cytoplasmic and SCV pH (Chakraborty et al., 2015). This drop in $\mathrm{pH}$ is crucial for induction of SPI-2 genes required for subsequent effector secretion. The effectors secreted by T3SS-SPI-2 change the early SCV into a late SCV that is uniquely suited for bacterial replication. Examples of SPI-2 effectors involved in SCV maturation include SifA and SopD2. SifA complexes with SifA-and-Kinesin-Interacting-Protein (SKIP). The SifA-SKIP complex sequesters and binds Rab9, thereby inhibiting Rab9-dependent recruitment of MPR (McGourty et al., 2012). SopD2 impairs the Rab7-dependent recruitment of RILP and FYCO1 (FYVE and Coiled-coil domain Containing protein 1). RILP and FYCO1 are involved in vesicular trafficking along microtubules and indirect inhibition of their recruitment by SopD2 delays delivery of the SCV to lysosomes (D'Costa et al., 2015). At this stage, the SCV is similar to a late endosome (with markers LAMP-1, Rab7 and V-ATPase), but not enriched with lysosomal enzymes, possibly due to the lack of MPR and incomplete lysosome fusion (McGourty et al., 2012). Similar to Chlamydia, Salmonella exploit dynein-mediated transport (via its effectors) to arrive at a juxtanuclear position near the microtubule organizing center (MTOC). At this location, Salmonella distinguishes itself from other intracellular pathogens with the formation of a dynamic tubular network composed of Salmonella induced filaments (SIFs) (Knuff and Finlay, 2017). SIFs are required for SCV integrity, enabling continuous fusion of host vesicles to SCV and are associated with late endosomal markers such as LAMPs, Rab7, V-ATPase, cholesterol and lysobisphosphatidic acid (LBPA), as well as low levels of MPR and cathepsin D. Furthermore, another similarity with other vacuole-living bacteria, is the communication between the SCV and the ER, illustrating the extensive interactions of SIFs/SCV with the host cell (Santos et al., 2015). However, unlike the other vacuolar bacteria's interaction with ER-derived components, the Salmonella SCV interaction with the ER-derived coat protein complex II (COPII) can result in SCV rupture and Salmonella hyper-replication in the cytosol (Santos et al., 2015).

In comparison to the more meticulous modulations mentioned, Listeria monocytogenes takes a relatively more radical (and perhaps destructive) approach to ensure intracellular survival. Manipulation of the clathrin-mediated endocytic pathway facilitates entry into non-phagocytic cells (Veiga and Cossart, 2005), whereas entry into macrophages is achieved via phagocytosis and initial engulfment of bacteria to form phagosomes. However, with the help of the cholesterol-dependent pore forming toxin listeriolysin-O (LLO), phagosome-lysosome fusion is disrupted via dysregulation of $\mathrm{pH}$ and calcium gradients across the phagosome membrane (Henry et al., 2006; Shaughnessy et al., 2006). Additionally, with the help of two phospholipases (PlcA and PlcB), LLO promotes escape of the bacteria from phagosomes into the cytosol (Mitchell et al., 2015; Smith et al., 1995). Once in the cytosol, bacteria undergo rapid growth and subsequently hijack the host's actin polymerization machinery to move within the cytosol and ultimately spread in a cell-to-cell manner (Mitchell et al., 2015; Skoble et al., 2017). Although not as intricate as Brucella and Legionella, Listeria is also capable of slow replication in macrophage vacuoles (instead of rapid cytosol replication) via the formation of spacious Listeria-containing phagosomes (SLAPs) (Birmingham et al., 2008). SLAP formation is dependent on LLO, but unlike phagosome rupture observed with cytosolic life, intermediate LLO expression is required for interference with phagosomal $\mathrm{pH}$, without phagosomal rupture. Bacteria containing SLAPs are LAMP- ${ }^{+}$, which indicates that these are endocytic compartments. However, no drop in $\mathrm{pH}$ is observed, due to LLO-mediated uncoupling of $\mathrm{pH}$ gradients across the membrane and prevention of lysosome fusion. Furthermore, SLAP formation is dependent on autophagy and is hypothesized to be triggered by the damage caused to phagosomes by LLO.

The opportunistic pathogen Cryptococcus neoformans (Cn) is also capable of infecting and replicating at high numbers in macrophages and may possibly utilize these phagocytes as shuttle for their dissemination across the blood brain barrier. An important virulence factor of $\mathrm{Cn}$ is its capsule, which ensures survival by protecting against phagocytic uptake and oxidative stress, once infiltrated into the host circulation (Zaragoza et al., 2008; Bojarczuk et al., 2016). However, phagocytosis can be triggered by direct recognition of $\mathrm{Cn}$ capsule components or indirectly via complement (Johnston and May, 2013). After $\mathrm{Cn}$ internalization by macrophages, it resides in phagosomes which mature into a phagolysosome, as usual. Interestingly, this microbe does not seem to radically modulate the phagosomal maturation process, but rather seems able to thrive at the lower $\mathrm{pH}$ of the maturing phagosome. Some early- and late-endosomal markers are present on these phagosomes, including EEA-1, Rab5, Rab11, MPR, LAMP-1 and cathepsins, with live $\mathrm{Cn}$ inducing premature removal of Rab5 and Rab11 from the $\mathrm{Cn}$ phagosome, which may influence phagosome acidification (Tucker and Casadevall, 2002; Davis et al., 2015; Smith et al., 2015). The phagolysosomes still acidify, but final $\mathrm{pH}$ is maintained slightly higher, at around 5.3 (vs. normal phagolysosome $\mathrm{pH}$ of $\sim 4.5$ ), which is the optimal $\mathrm{pH}$ for $\mathrm{Cn}$ growth. Additionally, damage to the phagolysosome membrane favors Cn survival and possibly contributes to the slight increase in $\mathrm{pH}$ observed with live $\mathrm{Cn}$ (Davis et al., 2015). Recently urease activity was found to influence phagosomal $\mathrm{pH}$, which through production of urease-derived ammonia can increases pH (Lerm et al., 2017; Fu et al., 2018). Furthermore, membrane damage to the phagolysosome results in permeabilization of the membrane and subsequent leakage of lysosomal enzymes 
(e.g., cathepsins), the loss of which may also increase survival of $\mathrm{Cn}$ within the phagolysosome (Wozniak and Levitz, 2008). Furthermore, the release of these enzymes, can result in activation of inflammasomes and subsequent cell death (Chen et al., 2015). It is clear that $\mathrm{Cn}$ is capable of modulating phagosome maturation to some extent, but the search for responsible effectors is still ongoing.

These studies illustrate how some pathogens manipulate the phagocytic process in seemingly divergent ways to reach an identical end goal of intracellular survival. In doing so they ensure their own propagation and dissemination to elicit disease. Importantly, in our opinion, this demonstrated susceptibility to manipulation of the phagocytic process supports the feasibility of drug delivery systems that harness one or more of the microbial strategies presented here. Although there is still much research to be done on the exact microbial effectors involved in manipulation of the endocytic pathway, the available literature can already be used to make informed decisions as to which effectors can be used in the development of autologous drug delivery systems.

In the context of a complete macrophage-based drug delivery system, the manipulation of the endocytic pathway for retention and protection of cargo is only the first step. The next step to consider in the development of an effective delivery system, is the expulsion of drug cargo from macrophage vehicles. To this end, the mechanisms used by microbes can again be mined and possibly exploited to achieve cargo expulsion.

\section{Expulsion From Host Cell}

Turning attention now to the expulsion phase, which is a vital requirement for pathogenic dissemination of microorganisms and which can be induced by either the infected host cell, or by the pathogen itself. Some microorganisms utilize host cell machinery to facilitate their escape, while others induce either accidental or intended host cell death, resulting in their release from the cell as a "side-effect." Many microorganisms have been identified to have the ability to egress via one or more methods and some effectors in this process have been identified. However, in terms of manipulation of egress through upregulation or elimination of these effectors, very little data is available and substantial experimental work is still required in this niche. This can be attributed, at least in part, to a large degree of redundancy. This degree of redundancy is also seen in the bacterial mechanisms employed to modulate phagosome maturation, which adds complexity to the process of identifying a controllable pathway. Below, we provide a summary of the current knowledge regarding microbial egress, with an integrated discussion of its potential for therapeutic application.

Probably the most obvious technique used, given the ability of many microbes to manipulate phagosomal $\mathrm{pH}$ for intracellular survival, is the manipulation of $\mathrm{pH}$ to induce host cell death. This technique has been described in some detail for Mtb, which stabilizes phagosomal $\mathrm{pH}$ at $\sim 6.2-6.5$ by interfering with the V-ATPase complex (Sturgill-Koszycki et al., 1994; Seto et al., 2011; Queval et al., 2017). This raised $\mathrm{pH}$ level is a pre-requisite for the ESX-1 dependent rupture of the phagosome (Simeone et al., 2015). The ESX-1 (T7SS) secretory system secretes two effector proteins, namely EsxA and EsxB, which form a heterodimer and are secreted by $\mathrm{Mtb}$ in a co-dependent manner (Renshaw et al., 2002). EsxA has membrane permeabilizing properties and EsxB is thought to act as a chaperone to prevent degradation and/or premature lytic activity (de Jonge et al., 2007; Zhang et al., 2016). EsxA effects phagosome rupture and escape to the cytosol, while being aided by the cell wall lipid phthiocerol dimycocerosates (PDIM) (Augenstreich et al., 2017; Quigley et al., 2017). This lipid has been proposed to primarily aid in phagosomal rupture, resulting in increased numbers of cytosolic bacteria - which in turn induces host cell necrosis and ultimately Mtb dissemination (Augenstreich et al., 2017; Quigley et al., 2017).

Other bacteria have also been described to escape through host cell membrane rupture resulting in cell death, albeit achieved by slightly different techniques. Brucella for example replicates within host cells, dissociating into two phenotypes, namely a smooth and a rough type. The rough phenotype has cytotoxic activity which breaks down the cellular membrane and is essential for bacterial dissemination (Pei et al., 2006, 2014). In this way, Brucella egress and dissemination is achieved through caspase-2 mediated cell death (Chen and He, 2009; Chen et al., 2011). Furthermore, this mode of cell death results in a pro-inflammatory response and recruitment of additional macrophages - that can be infected - in further aid of Brucella dissemination (Pei et al., 2014). It has, however, been proposed that Brucella can disseminate via cell-to-cell spread using an autophagy related mechanism (Starr et al., 2012; Smith et al., 2016). The final phase of Brucellosis intracellular life cycle is the formation of an aBCV which results from the engulfment of rBCVs into autophagosome-like structures via an autophagic process (Starr et al., 2008, 2012). This transformation to an aBCV is an essential prerequisite for bacterial egress via cell-to-cell spread (Starr et al., 2012; Smith et al., 2016). Interestingly, the VirB T4SS has been implicated in Brucella release via cell death and cell-to-cell spread, although the bacterial effectors have not been identified (Pei et al., 2008; Smith et al., 2016). The different modes of dissemination are possibly due to differences in experimental conditions, such as bacterial strains and cell lines used. Different bacterial strains may have different effectors or altered expression profiles that may result in different post-replicative outcomes (i.e., cell death or cell-to-cell spread) and different cell lines will also react differently to secreted effectors.

Legionella can also be placed in the category of intracellular pathogens that escape through macrophage cell death. Once a replicative LCV is established inside the macrophage, the bacteria converts to a replicative form and multiplies within the enclosed LCV. At high multiplicities of infection and subsequent termination of replication Legionella exhibit contact-dependent cytotoxicity, resulting in formation of pores in the host cell membranes (Kirby et al., 1998; Bachman and Swanson, 2001). Initially pores are formed within the phagosomal membrane (of the LCV), resulting in release of bacteria into the cytosol. The cytosolic bacteria are then able to form pores within the plasma membrane, resulting in osmotic lysis and release of bacteria (i.e., necrosis) (Kirby et al., 1998; Alli et al., 2000). The importance of the Dot/Icm secretory system of Legionella for 
pore-formation mediated lysis, and specifically that of the small inner membrane protein, IcmT, has been illustrated (Molmeret et al., 2002). Interestingly, in primary protozoan host cells, Legionella is capable of non-lytic release. The bacterial effectors LepA and LepB have been shown to play a role in manipulating the amoeba hosts exocytic pathway for dissemination (Chen et al., 2004, 2007). These effectors may also play a role in Legionella release from human cells or phagosome maturation and can potential form part of an artificial microbe drug delivery system.

Literature indicates that Salmonella exits cells via several mechanisms, including programmed cell death and flagella-facilitated escape. For example, the SPI-1 effector SipB can act by inducing caspase-1-dependent pyroptosis in macrophages (Hersh et al., 1999; Li et al., 2016). Briefly, SipB binds to and activates caspase-1, resulting in the cleavage of pro-IL1 $\beta$ and its secretion (Hersh et al., 1999; Li et al., 2016). While this inflammatory response should result in elimination of Salmonella, the over-activation during infection results in release of large amounts of bacteria capable of infecting naïve recruited cells. SipB is also able to induce apoptosis in a caspase-1 independent manner involving activation of caspase-2, -3, -6, and -8 (Jesenberger et al., 2000). In addition to apoptosis and pyroptosis, Salmonella is also able to induce oncosis in macrophages (Sano et al., 2007). Oncosis is associated with macrophage swelling resulting in cell death, with Salmonella-induced oncosis characterized by F-actin dissociation. Subsequently the flagellated Salmonella escapes from oncotic macrophages via flagellar movement (Sano et al., 2007).

Although the methods presented here will effectively release intracellular cargo, the associated host cell death may significantly contribute to tissue damage and secondary inflammatory damage, which may further delay recovery of patients. While certainly an option to consider for further development, specifically where cell death and increased inflammation would not be as detrimental (e.g., cancerous tissue), a more optimal solution in scenarios where minimization of inflammation - as well as the availability of functional macrophages - might be more critical, would be achieving release of drugs without the sacrifice of host cells.

Manipulation of the host cell's expulsion mechanics without killing the host has indeed been described for a few microbes, although much less information is available in this context. From the literature, it seems that only two egress methods have been described: direct spread into neighboring cells and expulsion into the extracellular environment. As briefly eluded to earlier, Listeria monocytogenes escapes from the host phagosome into the cytosol through activity of pore forming LLO (Alberti-Segui et al., 2007). However, complete escape from the host macrophage is aided by the effector responsible for modulation of the host actin polymerization machinery. Surface anchored actin assembly-inducing protein (ActA) interacts with the ARP2/3 complex to mediate actin polymerization on the bacterial surface, which in turn creates sufficient force to induce membrane protrusion and cell-to-cell spread (Skoble et al., 2017). The actin-propelled bacteria creating these membrane protrusions induce uptake into neighboring cells via a process called efferocytosis (Czuczman et al., 2014). Here, LLO damages the plasma membrane of protrusions, resulting in surface presentation of the inner membrane leaflet lipid, phosphatidylserine (PS) (Czuczman et al., 2014). The PS ${ }^{+}$ protrusions are recognized by the $\mathrm{T}$ cell immunoglobulin and mucin-domain containing protein 4 (TIM-4) on macrophages, which subsequently mediates the uptake of $\mathrm{PS}^{+}$protrusions (Czuczman et al., 2014). The bacteria may also be present in $\mathrm{PS}^{+}$vesicles, formed as a result of $\mathrm{Ca}^{2+}$ dependent membrane repair and scission of the initial $\mathrm{PS}^{+}$protrusion (Czuczman et al., 2014). Both $\mathrm{PS}^{+}$vesicles and protrusions are similarly taken up by neighboring cells via TIM-4. Listeria are one of the few phagocytically internalized microorganisms that allow host cell survival after escape. Thus, the processes regarding expulsion of Listeria is of great interest as target for manipulation or adoption in therapeutic drug delivery systems.

Chlamydia is also known to exit the host cell by extrusion, although it can also induce cell lysis (Hybiske and Stephens, 2007). These methods exhibit almost identical prevalence, but are markedly distinct and independent. The exact bacterial trigger facilitating either one or the other outcome has yet to be fully elucidated. Cell lysis is known to be protease and calcium dependant, and entails perforation of the inclusion body (Chlamydia-containing vacuole) and plasma membrane (Hybiske and Stephens, 2007). Chlamydia-infected host cell lysis has been suggested to be linked to microbe-associated apoptotic cell death, although minimal direct evidence exists in support of this notion (Perfettini et al., 2003). Regardless, the extrusion capability of Chlamydia from host cells without cell lysis is of greater interest here. With this technique, inclusion body extrusion requires actin polymerization, myosin II, RhoA and Neuronal Wiskott-Aldrich Syndrome Protein (N-WASP) (Hybiske and Stephens, 2007). The formation of an actin coat around the inclusion is correlated to extrusion out of the host cell. Host- and bacterium-derived factors play a role in the formation of the actin coat. For example, in humans, host-derived septins (GTP-binding proteins) form structures around the inclusion and co-localize with $\mathrm{F}$ actin, resulting in the formation of $\mathrm{F}$ actin fibers around the inclusion (Chin et al., 2012; Volceanov et al., 2014). This process facilitates normal extrusion of Chlamydia inclusions from host cells (Volceanov et al., 2014). Actin stabilization by jasplakinolide (actin polymerization agent) alone was reported to induce extrusion, which substantiates the role of septins in extrusion (Hybiske and Stephens, 2007). In terms of therapeutic application, this may suggest that the intervention achieved by Chlamydia on the host cell mechanics may be less detrimental to the host cell compared to other microbial exit strategies. In a therapeutic context, this may result in faster normalization of function in the host cell, which is much desired, as these host cells may then be able to participate in the normal inflammatory process that would be required for clean up after the drug has fulfilled its function.

Non-lytic release into the extracellular space has also been observed for Cryptococcus neoformans, albeit at low frequencies of $5-15 \%$ in vivo (Bojarczuk et al., 2016). Autophagy has been implicated in $\mathrm{Cn}$ intracellular lifestyle with components such as Atg-2a, -5, -9a, -12 and LC3 observed in close proximity to the 
Cn containing phagosome (Qin et al., 2011; Nicola et al., 2012). The effect of autophagy is dependent on opsonin, macrophage type and activation state (Nicola et al., 2012). Autophagy does seem to play a role in host defense against $\mathrm{Cn}$, with disruptions in autophagy affecting host fungistatic activity and fungal growth (Qin et al., 2011; Nicola et al., 2012). However, this is a double edged sword with autophagy also seemingly playing a role in $\mathrm{Cn}$ release. This is evident by the observation of Atg-5-knockout clones of J774.16 and RAW264.7 cells having reduced incidence of non-lytic exocytosis events (Qin et al., 2011; Nicola et al., 2012). This, along with the observation of LC3 surrounded cells outside macrophages, suggests a possible role of autophagy in non-lytic release of $\mathrm{Cn}$ (Nicola et al., 2012). Therefore, while autophagy aids in the host defense against $\mathrm{Cn}$, it also participates in the dissemination of $\mathrm{Cn}$ through non-lytic release. This eludes to a balance that must be maintained by the host with regard to autophagy, with either decreased fungistatic activity combined with decreased non-lytic release, or vice versa. In addition to the potential role of autophagy in the non-lytic release of $\mathrm{Cn}$, other factors can also play a role in this route of $\mathrm{Cn}$ dissemination. The increase in $\mathrm{pH}$ of the $\mathrm{Cn}$-containing phagosome results in increased occurrence of non-lytic release. Artificially increasing phagosomal $\mathrm{pH}$ results in increased expulsion of $\mathrm{Cn}$ and when compared to the in vivo situation, the damage caused by $\mathrm{Cn}$ to the phagosome could result in a similar $\mathrm{pH}$ increase (Nicola et al., 2011). Unfortunately, the $\mathrm{Cn}$ effectors responsible for non-lytic release have not yet been elucidated. However, in addition to the potential role of autophagy, the MAP extracellular receptor kinase 5 (ERK5) of host cells is implicated in the regulation of non-lytic release, with its inhibition resulting in increased release rates (Gilbert et al., 2017). Furthermore, actin polymerization also plays a role in release of $\mathrm{Cn}$ from the host (Johnston and May, 2010). In contrast to other pathogens such as Listeria and Chlamydia, actin polymerization inhibits $\mathrm{Cn}$ release through actin flashes on the $\mathrm{Cn}$ containing phagosome (Johnston and May, 2010). Although some information regarding the non-lytic release of $\mathrm{Cn}$ is available, it still remains poorly understood and the exact mechanism for this escape method is still elusive.

The body of research investigating intracellular pathogens and their host-interacting mechanisms is significant. However, specific information on the microbial effectors is still largely lacking, probably owing to the fact that the main focus of research was the prevention of these microbial actions, rather than full elucidation thereof for implementation. In addition, effectors secreted by invading microbial forces exhibit a large degree of built in redundancy, so that it is not surprising that the task of identifying specific roles for specific effectors has remained largely unaccomplished. Specifically, in terms of microbial expulsion, information is largely lacking, with only a handful of known microbial effectors. Considering this, clearly a new approach is required. The recent advancements in gene editing, heterologous expression, live cell imaging and -omics technologies may provide a more powerful platform from which to investigate the complex host-pathogen interaction and the effectors involved, especially in the context of expulsion from immune cell hosts.

\section{THE IMPOSSIBLE MADE POSSIBLE?}

From our review of the literature, we propose that most of the limitations of current drug delivery systems can be overcome by harnessing microbial strategies. We hypothesize that the synthetic microbe drug delivery system we describe here would (a) address poor drug-delivery to target tissue - especially at sites with low blood supply - (b) increase treatment efficacy with lower treatment doses and thereby (c) reduce adverse host reactions. A visual representation of the proposed system is provided in Figure 2.

In terms of the system we propose, we foresee two novel preparatory steps to be done in parallel. Firstly, monocytes should be isolated from peripheral blood collected from individuals with a requirement for drug delivery, to enable autologous re-infusion. These macrophages can then be propagated in culture, differentiating and polarizing them into M1 phenotype macrophages, as previously described (Mia et al., 2014), to achieve the phenotype known to be most capable of translocating

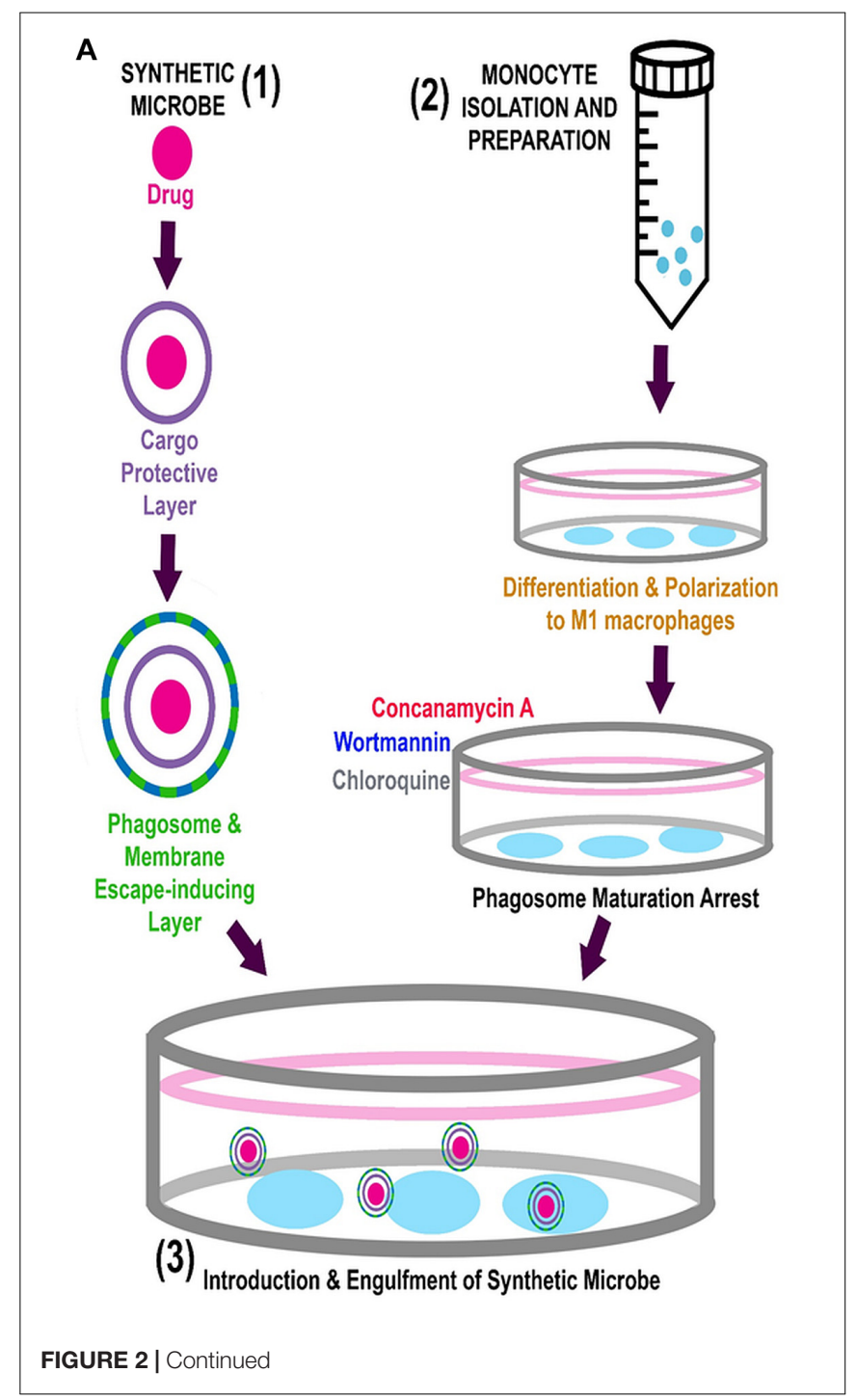




\section{B}

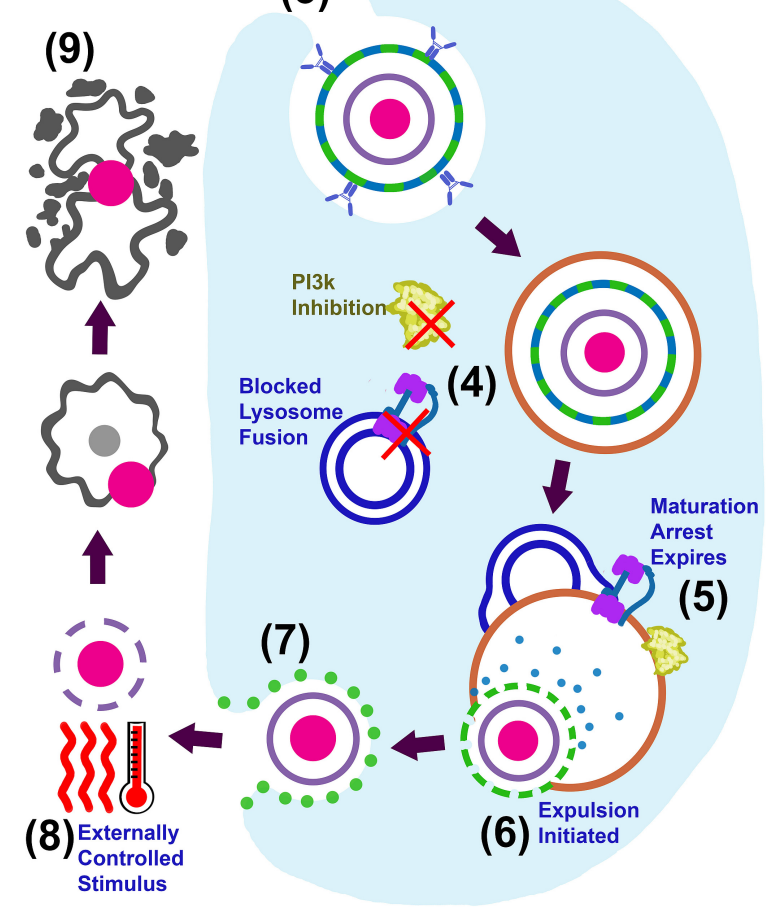

FIGURE 2 | Visual representation of the proposed system. (A) Simultaneous preparation of the synthetic microbes (1) and autologous macrophages (2) are followed by introduction of the synthetic microbe into phagosome maturation arrested macrophages (3). The complete system can now be administered into circulation for in vivo delivery. (B) Intracellular events for in vitro engulfment of synthetic microbe (3), in vivo maintenance/expulsion (4-7) and final delivery of drug at target site (8-9).

out of circulation and into tissue (Arnold et al., 2007; Africa and Smith, 2015a,b). In order to prevent intracellular degradation, polarized macrophages can be treated to achieve transient phagosome maturation arrest, by in vitro exposure to arresting agents such as Wortmannin, Concanamycin A and Chloroquine, which we have previously successfully illustrated (Visser and Smith, 2018). The fact that phagosome maturation arrest is achieved by in vitro intervention is a strength of this model, as this eliminates patient exposure to these potentially harsh chemicals and their related adverse effects. Secondly, the drug should be packaged in multiple layers of nanoparticle coatings (Figure 2A). These layers will serve to protect the drug from degradation, and can be peeled away in sequential, controlled steps to achieve controlled expulsion and release of the drug after delivery at its required site. With advancements in polymer sciences, smart polymers can be designed that have effectors conjugated to the polymers and can also be developed for directed multiphasic release of effectors at certain checkpoints. (The nature of the different layers will be clarified in the discussion of the expulsion phase and illustrated in Figure 2).

To construct the synthetic microbe drug delivery system, phagosome maturation arrested macrophages will engulf opsonized nanoparticle-enclosed drugs (the synthetic microbe) and form a phagosome containing this cargo (Figure 2). The number of synthetic microbes taken up per macrophage may be optimized by adjusting macrophage-microbe exposure time. Uptake is largely dependent on particle proximity to the macrophage as well as size and shape of particles (Beningo and Wang, 2002; Champion and Mitragotri, 2006; Underhill and Goodridge, 2012). Determining the exact onset of engulfment, following introduction of particles into cell media, is complex and may need optimization on a case-specific basis. Unpublished data from our group suggests initiation of engulfment to occur within $5 \mathrm{~min}$, with particles included at a 1:4 ratio with cells. From the literature, the last stage of phagosome maturation (phagolysosome biogenesis) occurs around $1 \mathrm{~h}$ after engulfment (Jahraus et al., 1998). Our implementation of an arbitrary exposure time of $1 \mathrm{~h}$ resulted in significant $4.5 \mu \mathrm{m}$ particle engulfment ( $>1$ particle per cell, in $\sim 70 \%$ of cells) by both arrested and untreated macrophages. Since macrophages are capable of repeated engulfment cycles (irrespective of arrest), carrier cells should be removed from drug-containing media and washed after the appropriate engulfment period, to prevent overloading of macrophages and potentially compromising their ability to traverse endothelial barriers, as well as eventual cell death (if particle load exceeds cytosol volume or steric hindrance causes membrane rupture during transmigration) (Champion and Mitragotri, 2006; Visser and Smith, 2018). At this point, the drug-loaded macrophages are ready for autologous re-administration into the patient via infusion into the peripheral circulation (Figure 2B).

As stated earlier, macrophage migration to sites for delivery will rely on the inherent macrophage capacity for chemotactic mobility. However, in vivo testing of the system will elucidate whether further optimization is required. Upon arrival at the site for delivery, controlled expulsion of the cargo and subsequent release of the drug will be achieved through multiple consequential steps. Specifically, the phagosome lysing and membrane escaping agents (i.e., microbial effectors) would be released upon arrival at target location. This can only be achieved after transient phagosome maturation has expired and phagolysosomal degradation agents activate this layer. This biphasic layer would, in turn, allow escape of cargo into the cytosol, as well as expulsion thereof into the extracellular environment through microbial effectors. The substantial amount of genome data currently available should enable relatively comprehensive genomic data mining for novel and existing microbial effectors. The use of genome mining in this context will only be useful if information (e.g., homology regions, structural information) about effectors are available. The use of techniques such as proteomics along with bioinformatic mining/analysis has been suggested as more useful tools in combination to identify effectors (Na et al., 2015; Cheng et al., 2017). For example, in recent studies, proteomics have been used to identify novel effectors in Salmonella. Although proteomics can be a useful tool in identification of effectors it is only the first step. Next would be to heterologously express the identified effectors. Several bacterial effectors have been expressed in heterologous systems with success (Churchill et al., 2005; Skoble et al., 2017; Weigele et al., 2017). Most notably, the Listeria 
effectors LLO and ActA have been recombinantly expressed, with recombinant LLO also being used for the effective delivery of several compounds to the cytosol of cells (Lee et al., 1996; Mandal and Lee, 2002; Provoda et al., 2003; Stier et al., 2005; Skoble et al., 2017). The ability to identify and express possible effectors creates the opportunity to manipulate the host endocytic pathway. However, these effectors need to be protected from degradation and secreted in a sequential manner to effectively modulate host processes.

In order to prevent asynchrony of final drug release and arrival of synthetic microbe at the target site (i.e., release of drug at inappropriate sites), an innocuous, bio-compatible layer, such as PNIPAAm (mentioned above), could be incorporated. This layer would be the inner most layer and protect the drug until controlled drug dissemination at the required site is achieved via external stimulus (e.g., temperature or ultrasound). In the case of a PNIPAAm layer, final drug dissemination can be achieved by temperature increase (Feng et al., 2014). In addition, microbubbles could also be incorporated as protective drug micelle, instead of PNIPAAm. These microbubbles would then be degraded by external ultrasound stimulation (Huang et al., 2015; Lv et al., 2016; Fan et al., 2018). This layer should ideally consist of non-biodegradable but bio-compatible constituents, to prevent drug release into circulation and exposure to off-target tissue. Furthermore, augmented host protection can be achieved by allowing the safe extrusion of intact drug-micelles through the urinary tract.

The redundancy employed in the aforementioned approach is another characteristic that could be adopted from the plethora of redundancy employed by microbes during immune evasion, in order to maximize control over drug delivery and minimize risk of undesired effects.

\section{POST-DELIVERY CLEARANCE OF THE SYSTEM}

Given the potential complication of polymer accumulation in vivo, or premature drug release, it is perhaps relevant to briefly discuss safety aspects of this system. The utilization of M1 macrophages would be advantageous as they would remain at target locations post-delivery and could potentially contribute to faster resolution of either primary inflammation (present due to the disease state) or secondary inflammation (required to clear bacterial debris after effective treatment). In support of this theory, the M1/M2 phenotype presents with some plasticity as tissue resident macrophages have been shown to change phenotype following appropriate effector exposure or systemic infection and activation by pathogens (Arnold et al., 2007; Mills, 2012). This low risk feature is the result of the non-lytic mechanisms employed to achieve expulsion, which is novel.

In terms of clearance of the components of the nanoparticle layers themselves, polymeric nanoparticles have demonstrated clearance rates of hours to several days (Alexis et al., 2008; Feng et al., 2014). Macrophages failing to reach their intended target sites may still release the encapsulated drug, but final dissemination of drugs at unintended sites is unlikely, due to the requirement for external stimulus. Thus, encapsulated drugs, polymeric debris and loaded macrophages should be efficiently cleared from the system, so that no long-term implications due to residual drugs or synthetic microbe components are expected.

Clearly, a model proposing quite significant paradigm shifts, such as the one we described here, requires substantial in vitro and in vivo testing and optimization by multiple laboratories. Given the requirement for in vivo tracking of cells, a zebrafish model may be the ideal choice for pilot studies, as the basic anatomy of the fish facilitates leukocyte migration tracking (Langenau et al., 2004) and this model has been employed in the context of macrophage migration (Wang et al., 2016; BarrosBecker et al., 2017). Additionally, in vivo tracking can also be achieved by permanent intracellular cell labeling and/or lasing techniques (Schubert et al., 2015) that would elucidate the exact location of carrier macrophages as well as their cargo over an extended period of time.

\section{CONCLUSION}

Intracellular pathogens have developed a unique arsenal of tools to evade the immune system and thrive within host cells. The modulation of host processes, specifically modulation of the endocytic pathway, not only make these pathogens difficult to treat and dangerous, but also provides the opportunity to utilize and modify their methods for human gain. If the right combination of these effectors are repurposed, they can be used to develop a macrophage-based delivery system for the transport and controlled delivery of therapeutic agents packaged into a "synthetic microbe" as described here, to significant benefit of patients currently struggling with diseases at non-accessible sites or those caused by multi-drug resistant pathogens.

The success of a host-derived or biological delivery system is dependent on a key understanding of how microbial effectors work and what combination would result in effective release without severe side-effects, keeping in mind that the effectors are indeed potent virulence factors. Advancements in molecular biology, -omics, bioinformatics and live cell imaging have resulted in the identification of effectors and their roles in the host-microbe/pathogen interaction, using and building on this information, effectors can be chosen that would result in the desired outcome. Utilizing synthetic biology and heterologous expression, effectors can be produced and tailored for specific functions. Although some developmental steps for the proposed synthetic microbe drug delivery model remains to be addressed in more detail, we believe that rapid development in e.g., polymer design and the aforementioned advancements in techniques that are used to characterize and tailor effectors, can be used to overcome these limitations in the very near future.

\section{AUTHOR CONTRIBUTIONS}

All authors listed have made a substantial, direct and intellectual contribution to the work, and approved it for publication. 


\section{REFERENCES}

Abbas, A. K., Lichtman, A. H., and Pillai, S. (2014). Basic Immunology: Functions and Disorders of the Immune System, 4th Edn. Philadelphia, PA: Elsevier Ltd.

Africa, L. D., and Smith, C. (2015a). Sutherlandia frutescens may exacerbate HIVassociated neuroinflammation. J. Negat. Results Biomed. 14:14. doi: 10.1186/ s12952-015-0031-y

Africa, L. D., and Smith, C. (2015b). Using a simulated blood-brain barrier to investigate potential modulators of HIV-1-associated neuro-inflammatory processes in vitro. J. Res. Biol. 5, 5-20.

Alberti-Segui, C., Goeden, K. R., and Higgins, D. E. (2007). Differential function of Listeria monocytogenes listeriolysin $\mathrm{O}$ and phospholipases $\mathrm{C}$ in vacuolar dissolution following cell-to-cell spread. Cell. Microbiol. 9, 179-195. doi: 10. 1111/j.1462-5822.2006.00780.x

Alexis, F., Pridgen, E., Molnar, L. K., and Farokhzad, O. C. (2008). Factors affecting the clearance and biodistribution of polymeric nanoparticles. Mol. Pharm. 5, 505-515. doi: 10.1021/mp800051m

Alli, O. A., Gao, L. Y., Pedersen, L. L., Zink, S., Radulic, M., Doric, M., et al. (2000). Temporal pore formation-mediated egress from macrophages and alveolar epithelial cells by Legionella pneumophila. Infect. Immunol. 68, 6431-6440. doi: 10.1128/IAI.68.11.6431-6440.2000

Arellano-Reynoso, B., Lapaque, N., Salcedo, S., Briones, G., Ciocchini, A. E., Ugalde, R., et al. (2005). Cyclic $\beta$-1,2-glucan is a brucella virulence factor required for intracellular survival. Nat. Immunol. 6, 618-625. doi: 10.1038/ ni1202

Arnold, L., Henry, A., Poron, F., Baba-Amer, Y., van Rooijen, N., Plonquet, A., et al. (2007). Inflammatory monocytes recruited after skeletal muscle injury switch into anti-inflammatory macrophages to support myogenesis. J. Exp. Med. 204, 1057-1069. doi: 10.1084/jem.20070075

Augenstreich, J., Arbues, A., Simeone, R., Haanappel, E., Wegener, A., Sayes, F., et al. (2017). ESX-1 and phthiocerol dimycocerosates of Mycobacterium tuberculosis act in concert to cause phagosomal rupture and host cell apoptosis. Cell. Microbiol. 19:e12726. doi: 10.1111/cmi.12726

Bachman, M. A., and Swanson, M. S. (2001). RpoS co-operates with other factors to induce Legionella pneumophila virulence in the stationary phase. Mol. Microbiol. 40, 1201-1214. doi: 10.1046/j.1365-2958.2001.02465.x

Baeck, C., Wehr, A., Karlmark, K. R., Heymann, F., Vucur, M., Gassler, N., et al. (2012). Pharmacological inhibition of chemokine CCL2 (MCP-1) diminishes liver macrophage infiltration and steatohepatitis in chronic hepatic injury. Gut 61, 416-426. doi: 10.1136/gutjnl-2011-300304

Bakowski, M. A., Braun, V., Lam, G. Y., Yeung, T., Do Heo, W., Meyer, T., et al. (2010). The Phosphoinositide phosphatase SopB manipulates membrane surface charge and trafficking of the Salmonella-containing vacuole. Cell Host Microbe 7, 453-462. doi: 10.1016/j.chom.2010.05.011

Bärlocher, K., Welin, A., and Hilbi, H. (2017). Formation of the legionella replicative compartment at the crossroads of retrograde trafficking. Front. Cell. Infect. Microbiol. 7:482. doi: 10.3389/fcimb.2017.00482

Barros-Becker, F., Lam, P., Fisher, R., and Huttenlocher, A. (2017). Live imaging reveals distinct modes of neutrophil and macrophage migration within intestinal tissues. J Cell Sci. 130, 3801-3808. doi: 10.1242/jcs.206128

Batrakova, E. V., Gendelman, H. E., and Kabanov, A. V. (2011). Cell-mediated drug delivery. Expert Opin. Drug Deliv. 8, 415-433. doi: 10.1517/17425247.2011. 559457

Becken, U., Jeschke, A., Veltman, K., and Haas, A. (2010). Cell-free fusion of bacteria-containing phagosomes with endocytic compartments. Proc. Natl. Acad. Sci. U.S.A. 107, 20726-20731. doi: 10.1073/pnas.100729 5107

Beningo, K. A., and Wang, Y. (2002). Fc-receptor-mediated phagocytosis is regulated by mechanical properties of the target. J. Cell Sci. 115, 849-856.

Betts-Hampikian, H. J., and Fields, K. A. (2010). The Chlamydial Type III secretion mechanism: revealing cracks in a tough nut. Front. Microbiol. 1:114. doi: 10. 3389/fmicb.2010.00114

Birmingham, C. L., Canadien, V., Kaniuk, N. A., Steinberg, B. E., Higgins, D. E., and Brumell, J. H. (2008). Listeriolysin O allows Listeria monocytogenes replication in macrophage vacuoles. Nature 451, 350-354. doi: 10.1038/ nature 06479

Blaudszun, A. R., Lian, Q., Schnabel, M., Loretz, B., Steinfeld, U., Lee, H. H., et al. (2014). Polyester-idarubicin nanoparticles and a polymer-photosensitizer complex as potential drug formulations for cell-mediated drug delivery. Int. J. Pharm. 474, 70-79. doi: 10.1016/j.ijpharm.2014.07.048

Bojarczuk, A., Miller, K. A., Hotham, R., Lewis, A., Ogryzko, N. V., Kamuyango, A. A., et al. (2016). Cryptococcus neoformans intracellular proliferation and capsule size determines early macrophage control of infection. Sci. Rep. 6:21489. doi: 10.1038/srep21489

Boschiroli, M. L., Ouahrani-Bettache, S., Foulongne, V., Michaux-Charachon, S., Bourg, G., Allardet-Servent, A., et al. (2002). The Brucella suis virB operon is induced intracellularly in macrophages. Proc. Natl. Acad. Sci. U.S.A. 99, 1544-1549. doi: 10.1073/pnas.032514299

Braun, V., Wong, A., Landekic, M., Hong, W. J., Grinstein, S., and Brumell, J. H. (2010). Sorting nexin 3 (SNX3) is a component of a tubular endosomal network induced by Salmonella and involved in maturation of the Salmonellacontaining vacuole. Cell. Microbiol. 12, 1352-1367. doi: 10.1111/j.1462-5822. 2010.01476.x

Bujny, M. V., Ewels, P. A., Humphrey, S., Attar, N., Jepson, M. A., and Cullen, P. J. (2008). Sorting nexin-1 defines an early phase of Salmonella-containing vacuole-remodeling during Salmonella infection. J. Cell Sci. 121, 2027-2036. doi: $10.1242 /$ jcs. 018432

Capmany, A., and Damiani, M. T. (2010). Chlamydia trachomatis intercepts golgi-derived sphingolipids through a rab14-mediated transport required for bacterial development and replication. PLoS One 5:e14084. doi: 10.1371/ journal.pone.0014084

Celli, J. (2006). Surviving inside a macrophage: the many ways of Brucella. Res. Microbiol. 157, 93-98. doi: 10.1016/j.resmic.2005.10.002

Celli, J. (2015). The changing nature of the Brucella-containing vacuole. Cell. Microbiol. 17, 951-958. doi: 10.1111/cmi.12452

Celli, J., de Chastellier, C., Franchini, D. M., Pizarro-Cerda, J., Moreno, E., and Gorvel, J. P. (2003). Brucella evades macrophage killing via VirB-dependent sustained interactions with the endoplasmic reticulum. J. Exp. Med. 198, 545-556. doi: 10.1084/jem.20030088

Chakraborty, S., Mizusaki, H., and Kenney, L. J. (2015). A FRET-based DNA biosensor tracks OmpR- dependent acidification of Salmonella during macrophage infection. PLoS Biol. 13:e1002116. doi: 10.1371/journal.pbio. 1002116

Champion, J. A., and Mitragotri, S. (2006). Role of target geometry in phagocytosis. Proc. Natl. Acad. Sci. U.S.A. 103, 4930-4934. doi: 10.1073/pnas.0600997103

Chang, Y. N., Guo, H., Li, J., Song, Y., Zhang, M., Jin, J., et al. (2013). Adjusting the balance between effective loading and vector migration of macrophage vehicles to deliver nanoparticles. PLoS One 8:e76024. doi: 10.1371/journal.pone.0076024

Chen, F., Ding, X., Ding, Y., Xiang, Z., Li, X., Ghosh, D., et al. (2011). Proinflammatory caspase-2-mediated macrophage cell death induced by a rough attenuated Brucella suis strain. Infect. Immunol. 79, 2460-2469. doi: 10.1128/IAI.00050-11

Chen, F., and He, Y. (2009). Caspase-2 mediated apoptotic and necrotic murine macrophage cell death induced by rough Brucella abortus. PLoS One 4:e6830. doi: 10.1371/journal.pone.0006830

Chen, J., de Felipe, K. S., Clarke, M., Lu, H., Anderson, O. R., Segal, G., et al. (2004). Legionella effectors that promote nonlytic release from protozoa. Science 303, 1358-1361. doi: 10.1126/science. 1094226

Chen, J., Reyes, M., Clarke, M., and Shuman, H. A. (2007). Host cell-dependent secretion and translocation of the LepA and LepB effectors of Legionella pneumophila. Cell. Microbiol. 9, 1660-1671. doi: 10.1111/j.1462-5822.2007. 00899.x

Chen, M., Xing, Y., Lu, A., Fang, W., Sun, B., Chen, C., et al. (2015). Internalized Cryptococcus neoformans activates the canonical Caspase-1 and the noncanonical Caspase-8 inflammasomes. J. Immunol. 195, 4962-4972. doi: 10.4049/jimmunol.1500865

Cheng, S., Wang, L., Liu, Q., Qi, L., Yu, K., Wang, Z., et al. (2017). Identification of a novel Salmonella Type III effector by quantitative secretome profiling. Mol. Cell. Proteomics 16, 2219-2228. doi: 10.1074/mcp.RA117.000230

Chin, E., Kirker, K., Zuck, M., James, G., and Hybiske, K. (2012). Actin recruitment to the Chlamydia inclusion is spatiotemporally regulated by a mechanism that requires host and bacterial factors. PLoS One 7:e46949. doi: 10.1371/journal. pone. 0046949

Churchill, R. L. T., Lee, H., and Hall, J. C. (2005). Rapid purification of recombinant listeriolysin O (LLO) from Escherichia coli. J. Ind. Microbiol. Biotechnol. 32, 355-363. doi: 10.1007/s10295-005-0002-2 
Comerci, D. J., Martínez-Lorenzo, M. J., Sieira, R., Gorvel, J. P., and Ugalde, R. A. (2001). Essential role of the VirB machinery in the maturation of the Brucella abortus-containing vacuole. Cell. Microbiol. 3, 159-168. doi: 10.1046/j.14625822.2001.00102.x

Curtis, L. M., and Gluck, S. (2005). Distribution of Rab GTPases in mouse kidney and comparison with vacuolar $\mathrm{H}^{+}$-ATPase. Nephron Physiol. 100, 31-42. doi: $10.1159 / 000085114$

Czuczman, M. A., Fattouh, R., van Rijn, J. M., Canadien, V., Osborne, S., Muise, A. M., et al. (2014). Listeria monocytogenes exploits efferocytosis to promote cell-to-cell spread. Nature 509, 230-234. doi: 10.1038/nature13168

Davis, M. J., Eastman, A. J., Qiu, Y., Gregorka, B., Kozel, T. R., Osterholzer, J. J., et al. (2015). Cryptococcus neoformans-induced macrophage lysosome damage crucially contributes to fungal virulence. J. Immunol. 194, 2219-2231. doi: 10.4049/jimmunol.1402376

D’Costa, V. M., Braun, V., Landekic, M., Shi, R., Proteau, A., McDonald, L., et al. (2015). Salmonella disrupts host endocytic trafficking by SopD2-mediated inhibition of Rab7. Cell Rep. 12, 1508-1518. doi: 10.1016/j.celrep.2015.07.063

de Almeida, A. C., Barbosa, S. M., de Lourdes Rios Barjas-Castro, M., Olalla-Saad, S. T., and Condino-Neto, A. (2012). IFN-beta, IFN-gamma, and TNF-alpha decrease erythrophagocytosis by human monocytes independent of SIRPalpha or SHP-1 expression. Immunopharmacol. Immunotoxicol. 34, 1054-1059. doi: 10.3109/08923973.2012.697470

de Jonge, M. I., Pehau-Arnaudet, G., Fretz, M. M., Romain, F., Bottai, D., Brodin, P., et al. (2007). ESAT-6 from Mycobacterium tuberculosis dissociates from its putative chaperone CFP-10 under acidic conditions and exhibits membranelysing activity. J. Bacteriol. 189, 6028-6034. doi: 10.1128/JB.00469-07

Doshi, N., Swiston, A. J., Gilbert, J. B., Alcaraz, M. L., Cohen, R. E., Rubner, M. F., et al. (2011). Cell-based drug delivery devices using phagocytosisresistant backpacks. Adv. Mater. 23, 105-109. doi: 10.1002/adma.2010 04074

Dou, H., Destache, C. J., Morehead, J. R., Mosley, R. L., Boska, M. D., Kingsley, J., et al. (2006). Development of a macrophage-based nanoparticle platform for antiretroviral drug delivery. Blood 108, 2827-2835. doi: 10.1182/blood-200603-012534

Dreaden, E. C., Mwakwari, S. C., Austin, L. A., Kieffer, M. J., Oyelere, A. K., and El-Sayed, M. A. (2012). Small molecule-gold nanorod conjugates selectively target and induce macrophage cytotoxicity towards breast cancer cells. Small 8, 2819-2822. doi: 10.1002/smll.201200333

Evans, M. A., Huang, P.-J., Iwamoto, Y., Ibsen, K. N., Chan, E. M., Hitomi, Y., et al. (2018). Macrophage-mediated delivery of light activated nitric oxide prodrugs with spatial, temporal and concentration control. Chem. Sci. 9, 3729-3741. doi: $10.1039 / \mathrm{c} 8 \mathrm{sc} 00015 \mathrm{~h}$

Fairn, G. D., and Grinstein, S. (2012). How nascent phagosomes mature to become phagolysosomes. Trends Immunol. 33, 397-405. doi: 10.1016/j.it.2012. 03.003

Fan, C. H., Lee, Y. H., Ho, Y. J., Wang, C. H., Kang, S. T., and Yeh, C. K. (2018). Macrophages as drug delivery carriers for acoustic phase-change droplets. Ultrasound Med. Biol. 44, 1468-1481. doi: 10.1016/j.ultrasmedbio.2018. 03.009

Feng, S., Cui, S., Jin, J., and Gu, Y. (2014). Macrophage as cellular vehicles for delivery of nanoparticles. J. Innov. Opt. Health Sci. 7:1450023. doi: 10.1142/ S1793545814500230

Ferrari, G., Langen, H., Naito, M., and Pieters, J. (1999). A coat protein on phagosomes involved in the intracellular survival of mycobacteria. Cell 97, 435-447. doi: 10.1016/S0092-8674(00)80754-0

Fratti, R. A., Backer, J. M., Gruenberg, J., Corvera, S., and Deretic, V. (2001). Role of phosphatidylinositol 3-kinase and Rab5 effectors in phagosomal biogenesis and mycobacterial phagosome maturation arrest. J. Cell Biol. 154, 631-644. doi: $10.1083 /$ jcb.200106049

Fu, M. S., Coelho, C., De Leon-Rodriguez, C. M., Rossi, D. C. P., Camacho, E., Jung, E. H., et al. (2018). Cryptococcus neoformans urease affects the outcome of intracellular pathogenesis by modulating phagolysosomal pH. PLoS Pathog. 14:e1007144. doi: 10.1371/journal.ppat.1007144

Gao, L. Y., and Abu Kwaik, Y. (1999). Activation of caspase 3 during Legionella pneumophila-induced apoptosis. Infect. Immunol. 67, 4886-4894.

Gaur, N. A., Hasek, J., Brickner, D. G., Qiu, H., Zhang, F., Wong, C. M., et al. (2013). Vps factors are required for efficient transcription elongation in budding yeast. Genetics 193, 829-851. doi: 10.1534/genetics.112.146308
Gerhardt, H., Walz, M. J., Faigle, M., Northoff, H., Wolburg, H., and Neumeister, B. (2000). Localization of Legionella bacteria within ribosomestudded phagosomes is not restricted to Legionella pneumophila. FEMS Microbiol. Lett. 192, 145-152. doi: 10.1111/j.1574-6968.2000.tb09373.x

Ghosh, S., and O'Connor, T. J. (2017). Beyond paralogs: the multiple layers of redundancy in bacterial pathogenesis. Front. Cell. Infect. Microbiol. 7:467. doi: $10.3389 /$ fcimb. 2017.00467

Gilbert, A. S., Seoane, P. I., Sephton-Clark, P., Bojarczuk, A., Hotham, R., Giurisato, E., et al. (2017). Vomocytosis of live pathogens from macrophages is regulated by the atypical MAP kinase ERK5. Sci. Adv. 3:e1700898. doi: 10. 1126/sciadv.1700898

Gnanadhas, D. P., Dash, P. K., Sillman, B., Bade, A. N., Lin, Z., Palandri, D. L., et al. (2017). Autophagy facilitates macrophage depots of sustained-release nanoformulated antiretroviral drugs. J. Clin. Invest. 127, 857-873. doi: 10.1172/ JCI90025

Gogulamudi, V. R., Dubey, M. L., Kaul, D., Atluri, V. S. R., and Sehgal, R. (2015). Downregulation of host tryptophan-aspartate containing coat (TACO) gene restricts the entry and survival of Leishmania donovani in human macrophage model. Front. Microbiol. 6:946. doi: 10.3389/fmicb.2015.0 0946

Guyton, C., and Hall, E. (2011). Medical Physiology, 12th Edn. Philadelphia, PA: Elsevier Ltd.

Henry, R., Shaughnessy, L., Loessner, M. J., Alberti-Segui, C., Higgins, D. E., and Swanson, J. A. (2006). Cytolysin-dependent delay of vacuole maturation in macrophages infected with Listeria monocytogenes. Cell. Microbiol. 8, 107-119. doi: 10.1111/j.1462-5822.2005.00604.x

Hersh, D., Monack, D. M., Smith, M. R., Ghori, N., Falkow, S., and Zychlinsky, A. (1999). The Salmonella invasin SipB induces macrophage apoptosis by binding to caspase-1. Proc. Natl. Acad. Sci. U.S.A. 96, 2396-2401. doi: 10.1073/pnas.96. 5.2396

Holden, C. A., Yuan, W. A., Lebman, D. A., and Yang, H. (2010). Surface engineering of macrophages with nanoparticles to generate a cell-nanoparticle hybrid vehicle for hypoxia-targeted drug delivery. Int. J. Nanomed. 5, 25-36.

Hong, P. C., Tsolis, R. M., and Ficht, T. A. (2000). Identification of genes required for chronic persistence of Brucella abortus in mice. Infect. Immun. 68, 41024107. doi: 10.1128/IAI.68.7.4102-4107.2000

Huang, W. C., Chiang, W. H., Cheng, Y. H., Lin, W. C., Yu, C. F., Yen, C. Y., et al. (2015). Tumortropic monocyte-mediated delivery of echogenic polymer bubbles and therapeutic vesicles for chemotherapy of tumor hypoxia. Biomaterials 71, 71-83. doi: 10.1016/j.biomaterials.2015.08.033

Hybiske, K., and Stephens, R. S. (2007). Mechanisms of host cell exit by the intracellular bacterium Chlamydia. Proc. Natl. Acad. Sci. U.S.A. 104, 1143011435. doi: 10.1073/pnas.0703218104

Jahraus, A., Storrie, B., Griffiths, G., and Desjardins, M. (1994). Molecular characterization of phagosomes. J. Cell Sci. 107, 145-157.

Jahraus, A., Tjelle, T. E., Berg, T., Habermann, A., Storrie, B., Ullrich, O., et al. (1998). In vitro fusion of phagosomes with different endocytic organelles. J. Biol. Chem. 273, 30379-30390. doi: 10.1074/jbc.273.46.30379

Jesenberger, V., Procyk, K. J., Yuan, J., Reipert, S., and Baccarini, M. (2000). Salmonella -induced Caspase-2 activation in macrophages. J. Exp. Med. 192, 1035-1046. doi: 10.1084/jem.192.7.1035

Johnston, S. A., and May, R. C. (2010). The human fungal pathogen Cryptococcus neoformans escapes macrophages by a phagosome emptying mechanism that is inhibited by Arp2 / 3 complex- mediated actin polymerisation. PLoS Pathog. 6:e1001041. doi: 10.1371/journal.ppat.1001041

Johnston, S. A., and May, R. C. (2013). Cryptococcus interactions with macrophages: evasion and manipulation of the phagosome by a fungal pathogen. Cell. Microbiol. 15, 403-411. doi: 10.1111/cmi.12067

Kinchen, J. M., Doukoumetzidis, K., Almendinger, J., Stergiou, L., ToselloTrampont, A., Sifri, C. D., et al. (2008). A pathway for phagosome maturation during engulfment of apoptotic cells. Nat. Cell Biol. 10, 556-566. doi: 10.1038/ ncb 1718

Kirby, J. E., Vogel, J. P., Andrews, H. L., and Isberg, R. R. (1998). Evidence for pore-forming ability by Legionella pneumophila. Mol. Microbiol. 27, 323-336. doi: 10.1046/j.1365-2958.1998.00680.x

Klyachko, N. L., Haney, M. J., Zhao, Y., Manickam, D. S., Mahajan, V., Suresh, P., et al. (2014). Macrophages offer a paradigm switch for CNS delivery of therapeutic proteins. Nanomedicine 9, 1403-1422. doi: 10.2217/nnm.13.115 
Knuff, K., and Finlay, B. B. (2017). What the SIF is happening-the role of intracellular Salmonella-induced filaments. Front. Cell. Infect. Microbiol. 7:335. doi: $10.3389 /$ fcimb.2017.00335

Kruger, M. J., Myburgh, K. M., and Smith, C. (2014). Contusion injury with chronic in vivo polyphenol supplementation: leukocyte responses. Med. Sci. Sports Exerc. 46, 225-231. doi: 10.1249/MSS.0b013e3182a4e754

Langenau, D. M., Ferrando, A. A., Traver, D., Kutok, J. L., Hezel, J. P. D., Kanki, J. P., et al. (2004). In vivo tracking of $\mathrm{T}$ cell development, ablation, and engraftment in transgenic zebrafish. Proc. Natl. Acad. Sci. U.S.A. 101, 7369-7374. doi: 10.1073/pnas.0402248101

LaRock, D. L., Chaudhary, A., and Miller, S. I. (2015). Salmonellae interactions with host processes. Nat. Rev. Microbiol. 13, 191-205. doi: 10.1038/nrmicro3420

Lee, H., Zhang, D., Wu, J., Otterbein, L. E., and Jin, Y. (2017). Lung epithelial cell-derived microvesicles regulate macrophage migration via MicroRNA17/221-induced Integrin $\beta 1$ recycling. J. Immunol. 99, 1453-1464. doi: 10.4049/ jimmunol.1700165

Lee, K., Oh, Y., Daniel, A., Swanson, J. A., and Portnoy, D. A. (1996). Delivery of macromolecules into cytosol using liposomes containing hemolysin from Listeria monocytogenes. J. Biol. Chem. 271, 7249-7252. doi: 10.1074/jbc.271.13. 7249

Lee, Y., Ryu, J. W., Chang, H., Sohn, J. Y., Lee, K. W., Woo, C. W., et al. (2010). In vivo $\mathrm{MR}$ evaluation of the effect of the CCR2 antagonist on macrophage migration. Magn. Reason. Med. 64, 72-79. doi: 10.1002/mrm.22409

Lerm, B., Kenyon, C., Schwartz, I., Kroukamp, H., de Witt, R., Govender, N. P., et al. (2017). First report of urease activity in the novel systemic fungal pathogen Emergomyces africanus: a comparison with the neurotrope Cryptococcus neoformans. FEMS Yeast Res. 17:fox069. doi: 10.1093/femsyr/ fox069

Li, Z., Zheng, Q., Xue, X., Shi, X., Zhou, Y., Da, F., et al. (2016). Pyroptosis of Salmonella typhimurium-infected macrophages was suppressed and elimination of intracellular bacteria from macrophages was promoted by blocking QseC. Sci. Rep. 6:37447. doi: 10.1038/srep37447

Liu, W., Xiao, X., Demirci, G., Madsen, J., and Li, X. C. (2013). The innate NK cells and macrophages recognize and reject allogeneic non-self in vivo via different mechanisms. J. Immunol. 188, 2703-2711. doi: 10.4049/jimmunol.1102997

Lv, Y., Hao, L., Hu, W., Ran, Y., Bai, Y., and Zhang, L. (2016). Novel multifunctional $\mathrm{pH}$-sensitive nanoparticles loaded into microbubbles as drug delivery vehicles for enhanced tumor targeting. Sci. Rep. 6:29321. doi: 10.1038/srep29321

Mallo, G. V., Espina, M., Smith, A. C., Terebiznik, M. R., Alemán, A., Finlay, B. B., et al. (2008). SopB promotes phosphatidylinositol 3-phosphate formation on Salmonella vacuoles by recruiting Rab5 and Vps34. J. Cell Biol. 182, 741-752. doi: $10.1083 /$ jcb.200804131

Mandal, M., and Lee, K. D. (2002). Listeriolysin O-liposome-mediated cytosolic delivery of macromolecule antigen in vivo: enhancement of antigen-specific cytotoxic T lymphocyte frequency, activity, and tumor protection. Biochim. Biophys. Acta 1563, 7-17. doi: 10.1016/S0005-2736(02)00368-1

McGourty, K., Thurston, T. L., Matthews, S. A., Pinaud, L., Mota, L. J., and Holden, D. W. (2012). Salmonella inhibits retrograde trafficking of mannose6-phosphate receptors and lysosome function. Science 338, 963-967. doi: 10. $1126 /$ science. 1227037

Mia, S., Warnecke, A., Zhang, X. M., Malmström, V., and Harris, R. A. (2014). An optimized protocol for human M2 macrophages using M-CSF and IL$4 /$ IL-10/TGF- $\beta$ yields a dominant immunosuppressive phenotype. Scand. J. Immunol. 79, 305-314. doi: 10.1111/sji.12162

Miller, M. A., Zheng, Y., Gadde, S., Pfirschke, C., Zope, H., Engblom, C., et al. (2015). Tumour-associated macrophages act as a slow-release reservoir of nanotherapeutic Pt(IV) pro-drug. Nat. Commun. 6:8692. doi: 10.1038/ncomms9692

Mills, C. D. (2012). M1 and M2 Macrophages: oracles of health and disease. Crit. Rev. Immunol. 32, 463-488. doi: 10.1615/CritRevImmunol.v32.i6.10

Mills, C. D., Ley, K., Buchmann, K., and Canton, J. (2015). Sequential immune responses: weapons of immunity. J. Inflamm. 7, 443-449. doi: 10.1159/ 000380910

Mitchell, G., Ge, L., Huang, Q., Chen, C., Kianian, S., Roberts, M. F., et al. (2015). Avoidance of autophagy mediated by PlcA or ActA is required for Listeria monocytogenes growth in macrophages. Infect. Immun. 83, 2175-2184. doi: 10.1128/IAI.00110-15

Molmeret, M., Alli, O. A. T., Zink, S., Flieger, A., Cianciotto, N. P., and Kwaik, Y. A. (2002). IcmT is essential for pore formation-mediated egress of Legionella pneumophila from mammalian and protozoan cells. Infect. Immun. 70, 69-78. doi: 10.1128/IAI.70.1.69-78.2002

Molmeret, M., Zink, S. D., Han, L., Abu-Zant, A., Asari, R., Bitar, D. M., et al. (2004). Activation of caspase-3 by the Dot/Icm virulence system is essential for arrested biogenesis of the Legionella-containing phagosome. Cell. Microbiol. 6, 33-48. doi: 10.1046/j.1462-5822.2003.00335.x

Moradin, N., and Descoteaux, A. (2012). Leishmania promastigotes: building a safe niche within macrophages. Front. Cell. Infect. Microbiol. 2:121. doi: 10.3389/ fcimb.2012.00121

Muthana, M., Giannoudis, A., Scott, S. D., Fang, H., Coffelt, S. B., Morrow, F. J., et al. (2011). Use of macrophages to target therapeutic adenovirus to human prostate tumors. Cancer Res. 71, 1805-1816. doi: 10.1158/0008-5472.CAN-102349

Na, H. N., Yoo, Y. H., Yoon, C. N., and Lee, J. S. (2015). Unbiased proteomic profiling strategy for discovery of bacterial effector proteins reveals that Salmonella protein PheA is a host cell cycle regulator. Chem. Biol. 22, 453-459. doi: 10.1016/j.chembiol.2015.03.008

Ng, E. L., Wang, Y., and Tang, B. L. (2007). Rab22B's role in trans-Golgi network membrane dynamics. Biochem. Biophys. Res. Commun. 361, 751-757. doi: 10. 1016/j.bbrc.2007.07.076

Nicola, A. M., Albuquerque, P., Martinez, L. R., Dal-Rosso, R. A., Saylor, C., De Jesus, M., et al. (2012). Macrophage autophagy in immunity to Cryptococcus neoformans and Candida albicans. Infect. Immun. 80, 3065-3076. doi: 10.1128/ IAI.00358- 12

Nicola, A. M., Robertson, E. J., Albuquerque, P., Derengowski, L., da, S., and Casadevall, A. (2011). Nonlytic exocytosis of Cryptococcus neoformans from macrophages occurs in vivo and is influenced by phagosomal $\mathrm{pH}$. mBio 2:e00167-11. doi: 10.1128/mBio.00167-11

Nimse, S. B., and Pal, P. (2015). Free radicals, natural antioxidants, and their reaction mechanisms. RSC Adv. 5, 27986-28006. doi: 10.1039/C4RA13315C

Oh, N., and Park, J.-H. (2014). Endocytosis and exocytosis of nanoparticles in mammalian cells. Int. J. Nanomed. 9(Suppl. 1), 51-63.

Owen, M. R., Byrne, H. M., and Lewis, C. E. (2004). Mathematical modelling of the use of macrophages as vehicles for drug delivery to hypoxic tumour sites. J. Theor. Biol. 226, 377-391. doi: 10.1016/j.jtbi.2003. 09.004

Pang, L., Qin, J., Han, L., Zhao, W., Liang, J., Xie, Z., et al. (2016). Exploiting macrophages as targeted carrier to guide nanoparticles into glioma. Oncotarget 7, 37081-37091. doi: 10.18632/oncotarget.9464

Patki, V., Lawe, D. C., Corvera, S., Virbasius, J. V., and Chawla, A. (1998). A functional PtdIns(3)P-binding motif. Nature 394, 433-434. doi: 10.1038/ 28771

Pei, J., Kahl-McDonagh, M., and Ficht, T. A. (2014). Brucella dissociation is essential for macrophage egress and bacterial dissemination. Front. Cell. Infect. Microbiol. 4:23. doi: 10.3389/fcimb.2014.00023

Pei, J., Turse, J. E., Wu, Q., and Ficht, T. A. (2006). Brucella abortus rough mutants induce macrophage oncosis that requires bacterial protein synthesis and direct interaction with the macrophage. Infect. Immun. 74, 2667-2675. doi: 10.1128/IAI.74.5.2667-2675.2006

Pei, J., Wu, Q., Kahl-McDonagh, M., and Ficht, T. A. (2008). Cytotoxicity in macrophages infected with rough Brucella mutants is type IV secretion system dependent. Infect. Immun. 76, 30-37. doi: 10.1128/IAI.00379-07

Perfettini, J. L., Hospital, V., Stahl, L., Jungas, T., Verbeke, P., and Ojcius, D. M. (2003). Cell death and inflammation during infection with the obligate intracellular pathogen, Chlamydia. Biochimie 85, 763-769. doi: 10.1016/j.biochi. 2003.08.006

Petersen, K., and Smith, C. (2016). Ageing-associated oxidative stress and inflammation are alleviated by products from grapes. Oxid. Med. Cell. Longev. 2016:6236309. doi: 10.1155/2016/6236309

Pizarro-Cerdá, J., Moreno, E., Sanguedolce, V., Mege, J. L., and Gorvel, J. P. (1998). Virulent Brucella abortus prevents lysosome fusion and is distributed within autophagosome-like compartments. Infect. Immun. 66, 2387-2392.

Provoda, C. J., Stier, E. M., and Lee, K. D. (2003). Tumor cell killing enabled by listeriolysin O-liposome-mediated delivery of the protein toxin gelonin. J. Biol. Chem. 278, 35102-35108. doi: 10.1074/jbc.M305411200

Qin, Q. M., Luo, J., Lin, X., Pei, J., Li, L., Ficht, T. A., et al. (2011). functional analysis of host factors that mediate the intracellular lifestyle of Cryptococcus neoformans. PLoS Pathog. 7:e1002078. doi: 10.1371/journal.ppat.1002078 
Queval, C. J., Song, O. R., Carralot, J. P., Saliou, J. M., Bongiovanni, A., Deloison, G., et al. (2017). Mycobacterium tuberculosis controls phagosomal acidification by targeting CISH-mediated signaling. Cell Rep. 20, 3188-3198. doi: 10.1016/j.celrep.2017.08.101

Quigley, J., Hughitt, V. K., Velikovsky, C. A., Mariuzza, R. A., El-Sayed, N. M., and Briken, V. (2017). The cell wall lipid PDIM contributes to phagosomal escape and host cell exit of Mycobacterium tuberculosis. mBio 8:e0148-17. doi: 10.1128/mBio.00148-17

Rai, M. N., Sharma, V., Balusu, S., and Kaur, R. (2015). An essential role for phosphatidylinositol 3-kinase in the inhibition of phagosomal maturation, intracellular survival and virulence in Candida glabrata. Cell. Microbiol. 17, 269-287. doi: 10.1111/cmi. 12364

Redpath, S., Ghaza, L. P., and Gascoigne, N. R. (2001). Hijacking and exploitation of IL-10 by intracellular pathogens. Trends Microbiol. 9, 86-92. doi: 10.1016/ S0966-842X(00)01919-3

Renshaw, P. S., Panagiotidou, P., Whelan, A., Gordon, S. V., Hewinson, R. G., Williamson, R. A., et al. (2002). Conclusive evidence that the major T-cell antigens of the Mycobacterium tuberculosis complex ESAT- 6 and CFP-10 form a tight, 1:1 complex and characterization of the structural properties of ESAT-6, CFP-10, and the ESAT-6*CFP-10 complex. Implications for pathogenesis and virulence. J. Biol. Chem. 277, 21598-21603. doi: 10.1074/jbc.M201625200

Roy, C. R., Berger, K. H., and Isberg, R. R. (1998). Legionella pneumophila DotA protein is required for early phagosome trafficking decisions that occur within minutes of bacterial uptake. Mol. Microbiol. 28, 663-674. doi: 10.1046/j.13652958.1998.00841.x

Ruytinx, P., Proost, P., Van Damme, J., and Struyf, S. (2018). Chemokine-induced macrophage polarization in inflammatory conditions. Front. Immunol. 9:1930. doi: 10.3389/fimmu.2018.01930

Rzomp, K. A., Scholtes, L. D., Briggs, B. J., Whittaker, G. R., and Scidmore, M. A. (2003). Rab GTPases are recruited to chlamydial inclusions in both a speciesdependent and species-independent manner. Infect. Immun. 71, 5855-5870. doi: 10.1128/IAI.71.10.5855-5870.2003

Samie, M. A., and Xu, H. (2014). Lysosomal exocytosis and lipid storage disorders. J. Lipid Res. 55, 995-1009. doi: 10.1194/jlr.R046896

Sano, G.-I., Takada, Y., Goto, S., Maruyama, K., Shindo, Y., Oka, K., et al. (2007). Flagella facilitate escape of Salmonella from oncotic macrophages. J. Bacteriol. 189, 8224-8232. doi: 10.1128/JB.00898-07

Santos, J. C., Duchateau, M., Fredlund, J., Weiner, A., Mallet, A., Schmitt, C., et al. (2015). The COPII complex and lysosomal VAMP7 determine intracellular Salmonella localization and growth. Cell. Microbiol. 17, 1699-1720. doi: 10. $1111 / \mathrm{cmi} .12475$

Schroeder, G. N. (2018). The toolbox for uncovering the functions of legionella Dot/Icm type IVb secretion system effectors: current state and future directions. Front. Cell. Infect. Microbiol. 7:528. doi: 10.3389/fcimb.2017.0 0528

Schubert, M., Steude, A., Liehm, P., Kronenberg, N. M., Karl, M., Campbell, E. C., et al. (2015). Lasing within live cells containing intracellular optical microresonators for barcode-type cell tagging and tracking. Nano Lett. 15, 5647-5652. doi: 10.1021/acs.nanolett.5b02491

Scidmore, M. A., Fischer, E. R., and Hackstadt, T. (2003). Restricted fusion of Chlamydia trachomatis vesicles with endocytic compartments during the initial stages of infection. Infect. Immun. 71, 973-984. doi: 10.1128/IAI.71.2.973-984. 2003

Sendide, K., Deghmane, A. E., Pechkovsky, D., Av-Gay, Y., Talal, A., and Hmama, Z. (2005). Mycobacterium bovis BCG attenuates surface expression of mature Class II molecules through IL-10-dependent inhibition of cathepsin S. J. Immunol. 175, 5324-5332. doi: 10.4049/jimmunol.175.8.5324

Seto, S., Matsumoto, S., Tsujimura, K., and Koide, Y. (2010). Differential recruitment of CD63 and Rab7-interacting-lysosomal-protein to phagosomes containing Mycobacterium tuberculosisin macrophages. Microbiol. Immunol. 54, 170-174. doi: 10.1111/j.1348-0421.2010.00199.x

Seto, S., Tsujimura, K., and Koide, Y. (2011). Rab GTPases regulating phagosome maturation are differentially recruited to mycobacterial phagosomes. Traffic 12 , 407-420. doi: 10.1111/j.1600-0854.2011.01165.x

Shaughnessy, L. M., Hoppe, A. D., Christensen, K. A., and Swanson, J. A. (2006). Membrane perforations inhibit lysosome fusion by altering $\mathrm{pH}$ and calcium in Listeria monocytogenes vacuoles. Cell. Microbiol. 8, 781-792. doi: 10.1111/j. 1462-5822.2005.00665.x
Shi, M., Zhang, Y., Liu, L., Zhang, T., Han, F., Cleveland, J., et al. (2016). MAP1S protein regulates the phagocytosis of bacteria and toll-like receptor (TLR) signaling. J. Biol. Chem. 291, 1243-1250. doi: 10.1074/jbc.M115.687376

Simeone, R., Sayes, F., Song, O., Gröschel, M. I., Brodin, P., Brosch, R., et al. (2015). Cytosolic access of Mycobacterium tuberculosis: critical impact of phagosomal acidification control and demonstration of occurrence in vivo. PLoS Pathog. 11:e1004650. doi: 10.1371/journal.ppat.1004650

Simonsen, A., Lippe, R., Christoforidis, S., Gaullier, J. M., Brech, A., Callaghan, J., et al. (1998). EEA1 links PI3K function to Rab5 regulation of endosome fusion. Nature 394, 494-498. doi: 10.1038/28879

Skoble, J., Welch, M. D., Skoble, J., Portnoy, D. A., and Welch, M. D. (2017). Three regions within ActA promote Arp2 / 3 complex-mediated actin nucleation and Listeria monocytogenes motility. J. Cell Biol. 150, 527-538. doi: 10.1083/jcb.150. 3.527

Smith, C., Kruger, M. J., Smith, R. M., and Myburgh, K. H. (2008). The inflammatory response to skeletal muscle injury. Sports Med. 38, 947-969. doi: 10.2165/00007256-200838110-00005

Smith, E. P., Miller, C. N., Child, R., Cundiff, J. A., and Celli, J. (2016). Postreplication roles of the Brucella VirB Type IV secretion system uncovered via conditional expression of the VirB11 ATPase. mBio 7:e01730-16. doi: 10. 1128/mBio.01730-16

Smith, G. A., Marquis, H., Jones, S., Johnston, N. C., Portnoy, D. A., and Goldfine, H. (1995). The two distinct phospholipases C of Listeria monocytogenes have overlapping roles in escape from a vacuole and cell-to-cell spread. Infect. Immun. 63, 4231-4237.

Smith, L. M., Dixon, E. F., and May, R. C. (2015). The fungal pathogen Cryptococcus neoformans manipulates macrophage phagosome maturation. Cell. Microbiol. 17, 702-713. doi: $10.1111 / \mathrm{cmi} .12394$

Starr, T., Child, R., Wehrly, T. D., Hansen, B., Hwang, S., López-Otin, C., et al. (2012). Selective subversion of autophagy complexes facilitates completion of the Brucella intracellular cycle. Cell Host Microbe 11, 33-45. doi: 10.1016/j. chom.2011.12.002

Starr, T., Ng, T. W., Wehrly, T. D., Knodler, L. A., and Celli, J. (2008). Brucella intracellular replication requires trafficking through the late endosomal/lysosomal compartment. Traffic 9, 678-694. doi: $10.1111 / j .1600-0854.2008 .00718 . x$

Steele-Mortimer, O., Méresse, S., Gorvel, J. P., Toh, B. H., and Finlay, B. B. (1999). Biogenesis of Salmonella typhimurium-containing vacuoles in epithelial cells involves interactions with the early endocytic pathway. Cell. Microbiol. 1, 33-49. doi: 10.1046/j.1462-5822.1999.00003.x

Stephens, R., Kalman, S., Mitchell, W., Marathe, R., Lammel, C., Fan, J., et al. (1999). Comparative genomes of Chlamydia pneumoniae and C. trachomatis. Nat. Genet. 21, 385-389.

Stier, E. M., Mandal, M., and Lee, K. D. (2005). Differential cytosolic delivery and presentation of antigen by listeriolysin O-liposomes to macrophages and dendritic cells. Mol. Pharm. 2, 74-82. doi: 10.1021/mp049 $896 \mathrm{v}$

Strahl, T., and Thorner, J. (2007). Synthesis and function of membrane phosphoinositides in budding yeast, Saccharomyces cerevisiae. Biochim. Biophys. Acta 1771, 353-404. doi: 10.1016/j.bbalip.2007.01.015

Sturgill-Koszycki, S., Schlesinger, P. H., Chakraborty, P., Haddix, P. L., Collins, H. L., Fok, A. K., et al. (1994). Lack of acidification in Mycobacterium phagosomes produced by exclusion of the vesicular proton-ATPase. Science 263, 678-681. doi: 10.1126/science.8303277

Tanei, T., Leonard, F., Liu, X., Alexander, J. F., Saito, Y., Ferrari, M., et al. (2016). Redirecting transport of nanoparticle albumin-bound paclitaxel to macrophages enhances therapeutic efficacy against liver metastases. Cancer Res. 76, 429-439. doi: 10.1158/0008-5472.CAN-15-1576

Tilney, L. G., Harb, O. S., Connelly, P. S., Robinson, C. G., and Roy, C. R. (2001). How the parasitic bacterium Legionella pneumophila modifies its phagosome and transforms it into rough ER: implications for conversion of plasma membrane to the ER membrane. J. Cell Sci. 114, 4637-4650.

Tucker, S. C., and Casadevall, A. (2002). Replication of Cryptococcus neoformans in macrophages is accompanied by phagosomal permeabilization and accumulation of vesicles containing polysaccharide in the cytoplasm. Proc. Natl. Acad. Sci. U.S.A. 99, 3165-3170. doi: 10.1073/pnas.052702799

Underhill, D. M., and Goodridge, H. S. (2012). Information processing during phagocytosis. Nat. Rev. Immunol. 12, 492-502. doi: 10.1038/nri3244 
Van Goethem, E., Poincloux, R., Gauffre, F., Maridonneau-Parini, I., and Le Cabec, V. (2018). Matrix architecture dictates three-dimensional migration modes of human macrophages: differential involvement of proteases and pod: some-like structures. J. Immunol. 184, 1049-1061. doi: 10.4049/jimmunol. 0902223

Veiga, E., and Cossart, P. (2005). Listeria hijacks the clathrin-dependent endocytic machinery to invade mammalian cells. Nat. Cell Biol. 7, 894-900. doi: 10.1038/ ncb 1292

Vergne, I., Chua, J., and Deretic, V. (2003). Mycobacterium tuberculosis phagosome maturation arrest: selective targeting of PI3P-dependent membrane trafficking. Traffic 4, 600-606. doi: 10.1034/j.1600-0854.2003.00120.x

Vergne, I., Chua, J., Lee, H. H., Lucas, M., Belisle, J., and Deretic, V. (2005). Mechanism of phagolysosome biogenesis block by viable Mycobacterium tuberculosis. Proc. Natl. Acad. Sci. U.S.A. 102, 4033-4038. doi: 10.1073/pnas. 0409716102

Vieira, O. V., Botelho, R. J., and Grinstein, S. (2002). Phagosome maturation: aging gracefully. Biochem. J. 366, 689-704. doi: 10.1042/bj20020691

Vieira, O. V., Bucci, C., Harrison, R. E., Trimble, W. S., Lanzetti, L., Gruenberg, J., et al. (2003). Modulation of Rab5 and Rab7 recruitment to phagosomes by phosphatidylinositol 3-kinase. Mol. Cell. Biol. 23, 2501-2514. doi: 10.1128/ MCB.23.7.2501-2514.2003

Vinet, A. F., Fukuda, M., Turco, S. J., and Descoteaux, A. (2009). The Leishmania donovani lipophosphoglycan excludes the vesicular proton-ATPase from phagosomes by impairing the recruitment of synaptotagmin. PLoS Pathog. 5:e1000628. doi: 10.1371/journal.ppat.1000628

Visser, J. G., and Smith, C. (2018). Development of a transendothelial shuttle by macrophage modification. J. Tissue Eng. Regen. Med. 12, e1889-e1898. doi: $10.1002 /$ term. 2620

Volceanov, L., Herbst, K., Biniossek, M., Schilling, O., Haller, D., Nölke, T., et al. (2014). Septins arrange F-actin-containing fibers on the Chlamydia trachomatis inclusion and are required for normal release of the inclusion by extrusion. mBio 5:e01802-14. doi: $10.1128 / \mathrm{mBio}$. 01802-14

Von Bargen, K., Gorvel, J. P., and Salcedo, S. P. (2012). Internal affairs: investigating the Brucella intracellular lifestyle. FEMS Microbiol. Rev. 36, 533-562. doi: 10. 1111/j.1574-6976.2012.00334.x

Walburger, A., Koul, A., Ferrari, G., Nguyen, L., Prescianotto-Baschong, C., Huygen, K., et al. (2004). Protein kinase G from pathogenic mycobacteria promotes survival within macrophages. Science 304, 1800-1804. doi: 10.1126/ science. 1099384

Wang, Q., Liu, S., Hu, D., Wang, Z., Wang, L., Wu, T., et al. (2016). Identification of apoptosis and macrophage migration events in paraquat-induced oxidative stress using a zebrafish model. Life Sci. 157, 116-124. doi: 10.1016/j.lfs.2016. 06.009

Weigele, B. A., Orchard, R. C., Jimenez, A., Cox, G. W., and Alto, N. M. (2017). A systematic exploration of the interactions between bacterial effector proteins and host cell membranes. Nat. Commun. 8:532. doi: 10.1038/s41467-01700700-7

Wong, D., Bach, H., Sun, J., Hmama, Z., and Av-Gay, Y. (2011). Mycobacterium tuberculosis protein tyrosine phosphatase (PtpA) excludes host vacuolar- $\mathrm{H}^{+}$. ATPase to inhibit phagosome acidification. Proc. Natl. Acad. Sci. U.S.A. 108, 19371-19376. doi: 10.1073/pnas.1109201108

Wozniak, K. L., and Levitz, S. M. (2008). Cryptococcus neoformans enters the endolysosomal pathway of dendritic cells and is killed by lysosomal components. Infect. Immunol. 76, 4764-4771. doi: 10.1128/IAI.00660-08

Xu, L., and Luo, Z. Q. (2013). Cell biology of infection by Legionella pneumophila. Microbes Infect. 15, 157-167. doi: 10.1016/j.micinf.2012.11.001

Yousefpour, P., and Chilkoti, A. (2014). Co-opting biology to deliver drugs. Biotechnol. Bioeng. 111, 1699-1716. doi: 10.1002/bit.25307

Zaragoza, O., Chrisman, C. J., Castelli, M. V., Frases, S., Cuenca-Estrella, M., Rodríguez-Tudela, J. L., et al. (2008). Capsule enlargement in Cryptococcus neoformans confers resistance to oxidative stress suggesting a mechanism for intracellular survival. Cell. Microbiol. 10, 2043-2057. doi: 10.1111/j.1462-5822. 2008.01186.x

Zhang, Q., Wang, D., Jiang, G., Liu, W., Deng, Q., Li, X., et al. (2016). EsxA membrane-permeabilizing activity plays a key role in mycobacterial cytosolic translocation and virulence: effects of single-residue mutations at glutamine 5 . Sci. Rep. 6:32618. doi: 10.1038/srep32618

Zhao, Y., Haney, M. J., Mahajan, V., Reiner, B. C., Dunaevsky, A., Mosley, R. L., et al. (2011). Active targeted macrophage-mediated delivery of catalase to affected brain regions in models of Parkinson's disease. J. Nanomed. Nanotechnol. S4:003. doi: 10.4172/2157-7439.S4-003

Conflict of Interest Statement: The authors declare that the research was conducted in the absence of any commercial or financial relationships that could be construed as a potential conflict of interest.

Copyright (c) 2019 Visser, Van Staden and Smith. This is an open-access article distributed under the terms of the Creative Commons Attribution License (CC BY). The use, distribution or reproduction in other forums is permitted, provided the original author(s) and the copyright owner(s) are credited and that the original publication in this journal is cited, in accordance with accepted academic practice. No use, distribution or reproduction is permitted which does not comply with these terms. 